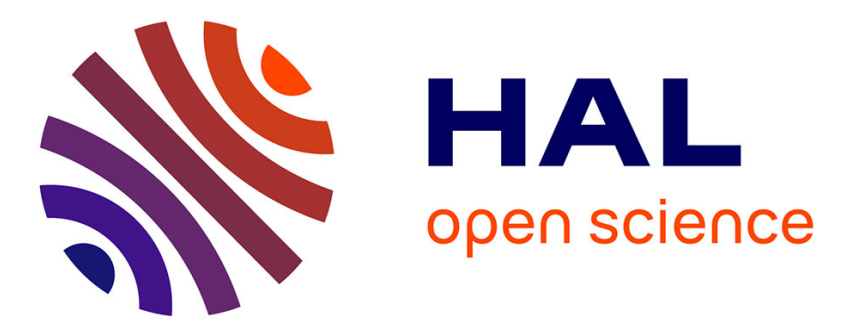

\title{
Innovation in gene regulation the case of chromatin computation
}

\author{
Sonja J. Prohaska, Peter F. Stadler, David C. Krakauer
}

\section{To cite this version:}

Sonja J. Prohaska, Peter F. Stadler, David C. Krakauer. Innovation in gene regulation the case of chromatin computation. Journal of Theoretical Biology, 2010, 265 (1), pp.27. 10.1016/j.jtbi.2010.03.011 . hal-00598092

\section{HAL Id: hal-00598092 \\ https://hal.science/hal-00598092}

Submitted on 4 Jun 2011

HAL is a multi-disciplinary open access archive for the deposit and dissemination of scientific research documents, whether they are published or not. The documents may come from teaching and research institutions in France or abroad, or from public or private research centers.
L'archive ouverte pluridisciplinaire HAL, est destinée au dépôt et à la diffusion de documents scientifiques de niveau recherche, publiés ou non, émanant des établissements d'enseignement et de recherche français ou étrangers, des laboratoires publics ou privés. 


\section{Author's Accepted Manuscript}

Innovation in gene regulation the case of chromatin computation

Sonja J. Prohaska, Peter F. Stadler, David C. Krakauer

PII: S0022-5193(10)00134-7

DOI: doi:10.1016/j.jtbi.2010.03.011

Reference: YJTBI 5912

To appear in: $\quad$ Journal of Theoretical Biology

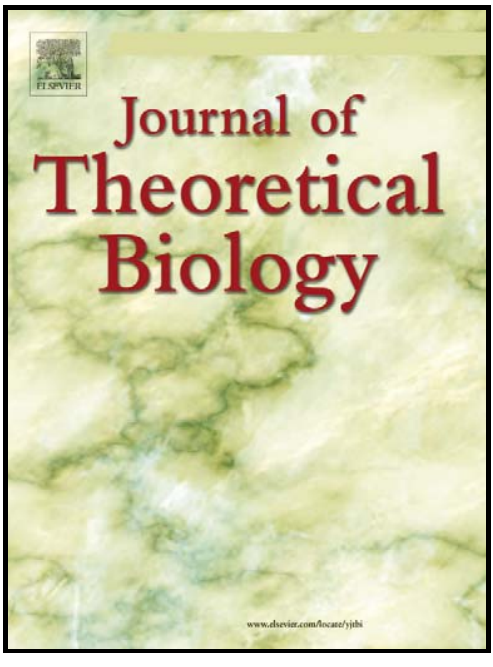

www.elsevier.com/locate/yjtb

Received date: 22 December 2009

Accepted date: 6 March 2010

Cite this article as: Sonja J. Prohaska, Peter F. Stadler and David C. Krakauer, Innovation in gene regulation the case of chromatin computation, Journal of Theoretical Biology, doi:10.1016/j.jtbi.2010.03.011

This is a PDF file of an unedited manuscript that has been accepted for publication. As a service to our customers we are providing this early version of the manuscript. The manuscript will undergo copyediting, typesetting, and review of the resulting galley proof before it is published in its final citable form. Please note that during the production process errors may be discovered which could affect the content, and all legal disclaimers that apply to the journal pertain. 


\title{
Innovation in Gene Regulation The Case of Chromatin Computation
}

\author{
Sonja J. Prohaska ${ }^{\mathrm{a}, \mathrm{b}}$, Peter F. Stadler ${ }^{\mathrm{a}, \mathrm{b}, \mathrm{c}, \mathrm{d}, \mathrm{e}}$, David C. Krakauer ${ }^{\mathrm{b}}$ \\ ${ }^{a}$ Bioinformatics Group, Department of Computer Science, and Interdisciplinary Center for Bioinformatics, University of Leipzig, Härtelstraße \\ 16-18, D-04107 Leipzig, Germany \\ ${ }^{b}$ Santa Fe Institute, 1399 Hyde Park Rd., Santa Fe, NM 87501, USA \\ ${ }^{c}$ Max-Planck Institute for Mathematics in the Sciences, Inselstraße 22, D-04103 Leipzig, Germany \\ ${ }^{d}$ Fraunhofer Institut für Zelltherapie und Immunologie - IZI, Perlickstraße 1, D-04103 Leipzig, Germany \\ ${ }^{e}$ Department of Theoretical Chemistry University of Vienna, Währingerstraße 17, A-1090 Wien, Austria
}

\begin{abstract}
Chromatin regulation is understood to be one of the fundamental modes of gene regulation in eukaryotic cells. We argue that the basic proteins that determine the chromatin architecture constitute an evolutionary ancient layer of transcriptional regulation common to all three domains of life. We explore phylogenetically, sources of innovation in chromatin regulation, focusing on protein domains related to chromatin structure and function, demonstrating a step-wise increase of complexity in chromatin regulation. Building upon the highly conserved use of variants of chromosomal architectural proteins to distinguish chromosomal states, Eukarya secondarily acquired mechanisms for "writing" chemical modifications onto chromatin that constitute persistent signals. The acquisition of reader domains enabled decoding of these complex, signal combinations and a decoupling of the signal from immediate biochemical effects. We show how the coupling of reading and writing, which is most prevalent in crown-group Eukarya, could have converted chromatin into a powerful computational device capable of storing and processing more information than pure cis-regulatory networks.
\end{abstract}

Key words: Chromatin, gene regulation, evolution

\section{Summary of Findings and Evolutionary Hypothesis}

Gene regulation in extant organisms is a complex adaptive system making use of a diversity of mechanisms to ensure that proteins and complementary macromolecular components are produced and maintained at functional levels in variable environments.

In this contribution we focus on one key regulatory subsystem of the cell: chromatin regulation. We argue that chromatin functioned as general regulator of transcription in the primordial nucleus, and that a series of key molecular innovations has significantly expanded the regulatory scope of the cell. In this section we summarize the key empirical results of the paper. Sections 2 through 6 provide the empirical support for these claims. In section 7, we formulate an evolutionary

Email addresses: sonja@bioinf .uni-leipzig.de (Sonja J. Prohaska), studla@bioinf .uni-leipzig.de (Peter F. Stadler), krakauer@santafe.edu (David C. Krakauer)

Preprint submitted to Preprint hypothesis that seeks to explain our findings in terms of the evolution of proto-genetic regulation. We then interpret this evolutionary sequence in terms of increasing computational power by locating key stages of the evolutionary sequence within a formal model of computation. Since sections 2-6 are primarily concerned with presenting evidence, those interested in the conceptual development of the argument can focus on sections 7 and 8.

Two variants of Chromosomal Architectural Proteins (ChAPs) are sufficient to define binary genomic, and potentially phenotypic, states. We show how extensions to this binary system lead to potential distinctions among an increasing number of genomic states, culminating in forms of control localized to specific sites in the genome, as illustrated for example by extant mammalian histone variants capable of differential expression and/or localization to restricted chromosomal regions [66].

The recruitment of pre-existing modification enDecember 22, 2009 
zymes able to modulate structural, or binding properties of ChAPs, represents a first significant regulatory innovation in chromatin. Modifications of ChAPs has been widely reported across the Archaea and Eukarya, with many of the modification enzymes homologous in both sequence and function. Modification enzymes are also present in bacteria but it is not known to what extent bacteria can instruct chemical changes to ChAPs.

The second innovation in chromatin regulation is the appearance of a diverse set of protein structures that bind with high specificity chemical modifications of histones. These modification "readers" are wide spread, among eukaryotes. The emergence of "readers" qualitatively changes chromatin regulation. Chemical modifications function as chromosomal marks or signals. Modification enzymes thereby shift from a purely structural role to an informational one, taking on the role of "writers" and "erasers" of signals association with gene expression.

Writing and reading allow for signal propagation (along the genome) and epigenetic transmission (across generations). Chromatin level modification also supports structural memory, a partial independence from the underlying genomic layer of information, and a capability to interpret sets of signals as inputs to generate complex outputs of chromatin modification. The extant epigenetic system observed in higher eukaryotes allows for fast and flexible/reversible integration of environmental signals into the regulatory program of the genome. These mechanisms provide crucial support for cell differentiation in multicellular organisms.

The addition of these key functions can be seen to be associated with increasing computational power, shifting from a simple markovian mechanism of regulation in the ancestral state, to a context sensitive system in several derived lineages, with capabilities perhaps exceeding those of sequence-mediated, cis-regulatory control.

\section{A Survey of Extant Chromatin Regulation}

\subsection{The Architecture of Chromatin}

DNA is never naked in a cell but associated with small, abundant, basic proteins, the ChAPs, that facilitate DNA organization and prevent DNA aggregation and tangling. This ensures efficient replication, segregation and gene expression. ChAPs are phylogenetically diverse $^{1}$. The tight association of DNA and ChAPs is

${ }^{1}$ Groups of unspecific DNA binding proteins, such as singlestranded DNA binding proteins or RecA are usually not counted as ChAPs. known as chromatin, and occurs in different compositions in all three domains of life [172, 104]. The ChAPs with the widest phylogenetic distribution are HU and histones in the Eubacterial and Eukaryotic clades, Archaea favor two types of ChAPs from a set comprising HU, Alba, and histone [104] summarized in Tab. 1. In addition to these common proteins, many nucleoidassociated proteins with more restricted phylogenetic distributions are known from microbes [146].

Histone proteins contain the histone fold, a 64 amino acid long helix-loop-helix-loop-helix motif stabilized through dimerization with a second histone fold. Functional characteristics distinguish DNA packaging via histones from other architectural proteins. In histone containing species, DNA is wrapped around a histone complex, forming the nucleosome leading to a significant reduction of the contour length of DNA. First identified in Euryarchaeota, representatives of archaeal histones have subsequently been found in species from all major archaeal lineages [36, 158]. Most archaeal genomes encode one to six distinct histone proteins [146, 8]. Many Crenarchaea, however, completely lack histones. The only ChAP that is highly conserved in this phylogenetic group is Cren7 [65]. Archaeal nucleosomes consist of an histone tetramer with about $60 \mathrm{bp}$ of DNA wrapped around the histones approximately once. Eukaryotic nucleosomes consist of a histone octamer in which two H3-H4 heterodimers form a histone core homologous to the archaeal histone tetramer [147]. In comparison to archaea, about twice as much DNA is wrapped around the octamer in two turns rather than one. Whereas archaeal histones introduce positive or negative supercoiling depending on salt concentration, temperature, and tetramer formation [106], eukaryotic nucleosomes always induce negative supercoiling (Tab. 1). Restricted to Eukaryotes are higher-order chromatin structures formed due to interactions between histone tails that protrude from the core and H1 (histone 1) - a non-histone fold linker protein. Linker histones in the protein configuration of Metazoans, appear first in late protists [84]. Dinoflagellates have secondarily lost their histones and do not form nucleosomes [115]. Their major ChAP, HCC, is most closely related to bacterial HU proteins [188].

Eubacteria do not as a rule have proteins with histonefolds [137]. An exception are the homologs of the nonhistone-fold protein histone $\mathrm{H} 1$ in Chlamydiae [64, 119] likely acquired by lateral transfer from a eukaryotic host. HU and its homologs are phylogenetically the most widespread ChAPs in Eubacteria. On average, three HU variants per genome are found, Tab. 1, which encode monomers that form homo- and heterodimers 
Table 1: Comparison of the main features of Chromosomal Architectural Proteins (ChAP) and their interaction with DNA in the three domains of life.

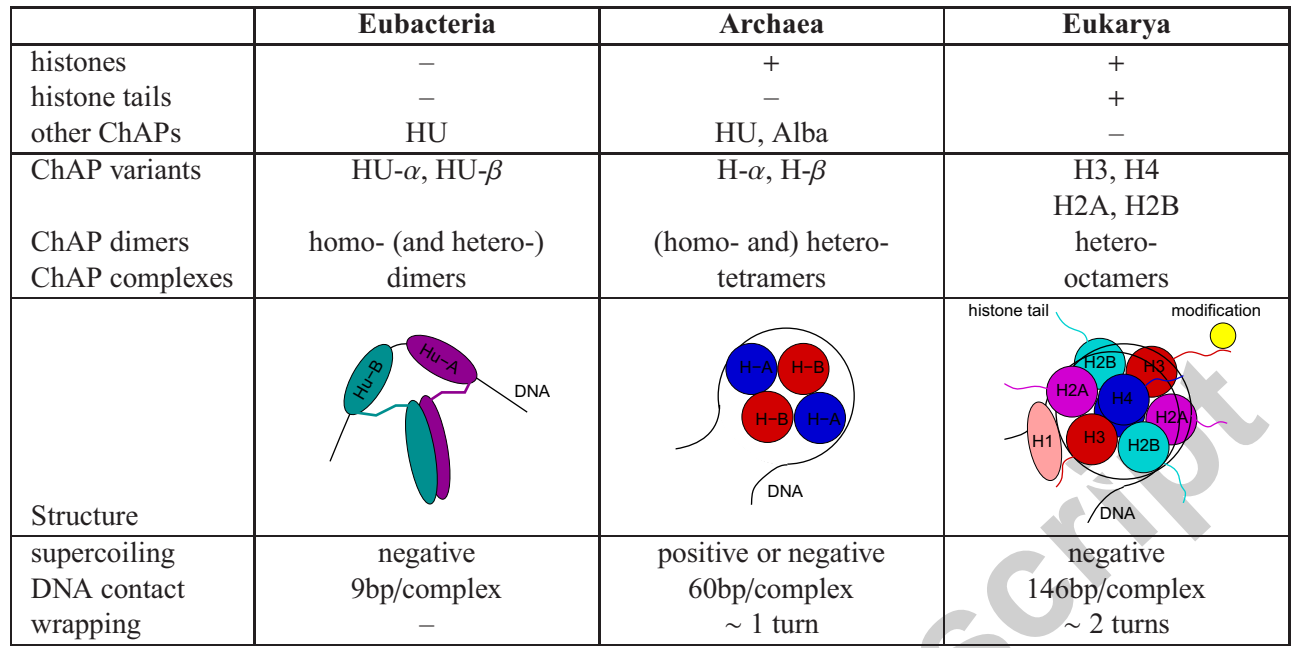

[132]. HU binds the DNA backbone in a sequenceunspecific manner, sharply bending DNA introducing negative supercoils. In $E$. coli, $\mathrm{HU}$ dimers bind on average every $290 \mathrm{bp}$ but form dense arrays with a dimer every $9 b p$ [27]. Surprisingly, HU is not essential for viability [44], as it can be replaced by alternative ChAPs [7]. To-date, HU homologs have been characterized in a few Archaea [126], and in a variety of eukaryotes including Giardia lamblia [174], Dinoflagellates [188] and Apicomplexa [5].

Alba ("acetylation lowers binding affinity") is a candidate non-histone ChAP $[17,181]$. It was first found in Archaea, where it is one of the most abundant proteins in thermophilic and hyperthermophilic species. Eukaryotic homologs in human, green plants, and protists have since been reported. Depending on its concentration and conformation, the Alba either bridges two DNA duplexes or cooperatively binds to a single DNA duplex [124]. Alba also binds to ssDNA and RNA in vivo [65], leading some to question it's role as a chromatin architectural protein.

In Eubacteria, binding by alternative ChAPs leads to different supercoiling patterns of DNA and to different spatial organizations of the nucleoid [169]. The binding affinities of ChAPs differ by at least an order of magnitude, exhibit varying levels of sequence specificity and can be sensitive to DNA curvature [7].

A fundamental difference between the chromatin organization of Eubacteria and the Eukarya/Archaea clade is that the latter two domains form multimeric nucle- osomes that have DNA wrapped around themselves, whereas eubacterial DNA is organized into loops with dense regions in complex with ChAPs. The complexes of DNA and ChaPs are referred to as nucleoid in both Archaea and Eubacteria despite structural differences.

\subsection{ChAP Variants}

ChAPs are frequently present in paralogous copies. The number of ChAP variants is limited in prokaryotes (on average 1 to 3 ), whereas eukaryotic genomes can encode a large numbers of differentially expressed paralogs [105].

Different cellular forms of microbes typically correlate with different nucleoid structures and differential abundance of the various nucleoid proteins [172]. As with $\mathrm{HU}$, the abundance of certain histone proteins changes with cell states in Archaea and Eukarya. Paralogs can influence the degree of compaction introduced into DNA [146]. H2A.Z, for example, is less stable than $\mathrm{H} 2 \mathrm{~A}$ and inhibits spreading of silent chromatin. Several paralogs/variants exist for all histone types in Eukarya. Phylogenetic analysis suggests that $\mathrm{H} 3$ and $\mathrm{H} 2 \mathrm{~A}$ are more variable than $\mathrm{H} 4$ and $\mathrm{H} 2 \mathrm{~B}$, respectively $[105,170]$.

Functional diversification has been demonstrated for all variants except H4. Depositioning of these proteins is dependent on the cell cycle (replication-dependent and replication-independent histone variants, $\mathrm{H} 3$ and H3.3 respectively) and cell type (sperm- and testis specific variants of $\mathrm{H} 1$ and $\mathrm{H} 3$ ). Alternative variants local- 
ize to genomic regions $[2,25,83,105,184]$, e.g. CENP$\mathrm{A}$, a H3 variant, and $\mathrm{MacroH} 2 \mathrm{~A}$ are deposited at centromers and the inactive $\mathrm{X}$ chromosome, respectively. $\mathrm{Hv} 1$ and $\mathrm{Hv} 2$ are $\mathrm{H} 2 \mathrm{~A}$ and $\mathrm{H} 3$ variants exclusively used in the macronucleus of Tetrahymena thermophila. Trypanosomes use specific combinations of numerous histone variants to ensure demarcation of polycistronic transcription units [156]. In addition, ChAPs can deviate from non-specific DNA binding, and binding preferences of each variant can diverge. Nucleosomes, for example, have a slight sequence preference $[9,101]$, favoring DNA that bends more easily. Furthermore, they tend to influence the positioning of neighboring nucleosomes [136]. Paralogous ChAPs are known to modulate the sequence preference for nucleosome positioning $[8,106]$.

A similar diversification of ChAPs is observed in Archaea and Eubacteria. Some Archaea have two histone variants, $\mathrm{H}-\alpha$ and $\mathrm{H}-\beta$. $\mathrm{H}-\beta$ has been shown to induce greater compaction [62]. Heterodimers occur predominantly in the stationary growth phase, whereas the $(\mathrm{H}-\alpha)_{2}$ homodimers are prevalent during exponential growth phase [145]. Alba often appears in two paralogs, with heterodimers promoting DNA compaction as with histone variants [80].

In $E$. coli and related enteric bacteria, the two $\mathrm{HU}$ variants $\alpha$ and $\beta$ form homo- and heterodimers. $\alpha \beta$ and $\alpha_{2}$ dominates the exponential growth phase. A shift to higher $\beta$ concentrations leads to formation of mainly $\alpha \beta$ and stationary phase [34].

\section{The Regulation of Transcription by Chromatin}

Chromatin structure has an immediate effect on local transcriptional activity. In Eubacteria, distinct nucleoid structures are associated with different cellular forms, such as vegetative cells or spores. At the onset of the stationary phase, bacterial chromatin undergoes a massive reorganization into ordered toroidal structures through a process dictated by the intrinsic properties of DNA and the ubiquitous, starvation-induced DNAbinding protein Dps [58]. Eubacteria appear to have evolved a hierarchy of nucleoid-associated factors with each spanning a different range of sequence specificity: less specific variants act as functional backups for the more specific variants $[131,7]$. Non-linear DNA structures promote signal integration paralleling the transduction cascades employed by higher organisms to control cell growth and differentiation [131]. Eubacterial ChAPs such as H-NS, FiS, IHF, and SLpA, therefore, act as general regulators of transcription [39, 130, 103].
DNA occupied by nucleosomes, and structured into chromatin blocks, slows down initiation and elongation of transcription compared to naked DNA $[6,79]$. This effect is strongest in eukaryotic octamers. However, for Eukaryotic $(\mathrm{H} 3-\mathrm{H} 4)_{2}$ tetramers as well as archaeal nucleosomes, DNA wrapped around histone tetramers is comparatively easy to access [147]. For initiation of transcription, activators usually bind the unoccupied "linker" DNA between nucleosomes [136]. Binding sites covered by a nucleosome are largely inaccessible to binding factors unless nucleosomes are destabilized, moved or evicted. Chromatin is understood as a negative regulator of transcription. A major distinction is made between open and closed, active and silent chromatin. Open chromatin correlates with active gene expression [143]. Nevertheless, chromatin opening and gene activation are distinct processes [148]. Closed chromatin, on the other hand, does not necessarily impair binding of transcription factors [143]. In fact, transcription factors are able to open chromatin [99, 33]. Reduced transcriptional activity is often associated with compacted "closed" nucleoid structures in Eubacteria [58].

Hence chromatin does not exclusively determine the onset or efficiency of transcription in most species. Only trypanosomatids rely largely on chromatin, and in particular, on variations in nucleosome compositions, $[162,156]$ to regulate gene epxression. In nearly all organisms, the local chromatin composition sets the stage for further, more specific mechanisms of regulation, such as sequence-specific transcription factors [97]. ChAPs therefore (nearly universally) act as general regulators of transcription.

\section{Chemical (De-)Modifications of ChAPs in the Three Domains of Life}

Chromatin destabilization and subsequent transcriptional activation can be achieved either by the exchange of ChAP variants, or by chemical modifications of ChAPs. As we have described above, the first mechanism is present in all three domains of life. By contrast, chemical modifications of ChAPs do not appear uniformly. We have performed a comprehensive evaluation of the phylogenetic distribution of key modification enzymes making use of the SCOP and SuperFamily databases [4, 186], and complemented these findings with an extensive literature survey of the topic (see Section 10). The results are summarized in Fig. 1 (see also Supplementary Tables) and commented upon in the following subsections. Overall, chemical modifications of 
ChAPs are prevalent in Archaea and Eukarya. In Eubacteria, only a few reports assert that phosphorylation [35] and hydroxybutyrylation [125] are present in this domain.

Chemical modifications of ChAPs can function in two fundamentally different ways: (1) by means of direct influence on the thermodynamic stability of chromatin, and (2) by means of active disassembly, eviction, or mobilization performed by enzyme complexes $[73,95]$.

In Archaea, the first, thermodynamic mode is predominant. For example, Alba - which stands for "acetylation lowers binding affinity" - is acetylated and deacetylated to decrease and increase the binding affinity of Alba to DNA. A regulatory role for direct chemical effects can also be observed in Eukarya. Acetylation of histone H3 and H4 in Eukarya leads to chromatin opening/activation [141]. As demonstrated for yeast, the level of gene expression is dependent on the total number of acetyl groups on H4K5, H4K8 and H4K12, whereas the sites themselves are interchangeable [43]. This observation of degeneracy has been seen as a challenge to the idea of a complex, chromatin code [72].

Several unrelated protein families are responsible for each type of ChAP modification. In the following sections, we examine phosphorylation, acetylation, methylation, and ubiquitination in some detail. In the presence of modification "readers", i.e. in the eukaryotes, we refer to modifiers functionally as "writers" and "erasers" describing the addition and removal of chemical modifications.

\section{1. (De-)Phosphorylation}

Protein phosphorylation involves chemical modifications to serine/threonine/tyrosine and histidine residues. The corresponding enzymes performing these reactions can be partitioned in histidine and serine/threonine/tyrosine protein kinases. The latter constitute a part of one protein superfamily and are the most common and abundant modification enzymes throughout all domains of life contributing to histone phosphorylation. Histidine phosphorylation on histones has also been reported for $\mathrm{H} 4$ in mammalian tissues [20, 163]. Phosphate groups can induce conformational changes and are used to activate enzymes and serve as signals. Phosphorylation cascades provide rapid response kinetics (e.g. heat shock response) and reversible action. Determining which from Ser/Thr/Tyr kinases phosphorylate ChAPs is difficult to estimate since reported histone kinases (e.g. Aurora, Polo, Nek, or Haspin [38]) can not be distinguished from other protein kinases based on family-level domain descriptions.
Phosphorylation of serine, threonine and tyrosine is reversed by phosphatases utilizing different protein domains and molecular mechanisms. In a phylogenetic respect, phosphatases co-occur with kinases. Nevertheless, genomes contain fewer phosphatases than kinases (in Eukarya by a factor of 10, Fig. 1) [85, 86]. This suggests that a highly specific phosphorylation machinery might be counteracted by a fairly general dephosphorylation machinery.

\section{2. (De-)Acetylation}

Protein lysine residues can be modified by acetylation or methylation. In general, acetylation of histones is known to correlate with transcriptional activity. However, certain sites, such as H4K16 in yeast, contradict this remark and seem to be strongly context dependent [43]. Eukaryotic HATs (histone acetyltransferases) belong to the (super)family of NATs (AcetylCoA N-acetyltransferases) among which are relatives of GCN5 and Elp3, MYST, p300/CBP, $\mathrm{TAF}_{I I} 250$ and nuclear hormone-related HATs [127]. NAT members not only perform protein/histone acetylation. Bacterial members of this superfamily can acetylate antibiotics [177]. Pats (protein acetyltransferases) from Eubacteria, serve as regulators of metabolic enzymes [160] and might have been co-opted into the role of specific regulators of Alba within Archaea [107]. According to our analysis, NAT-domain containing acetyltransferases are phylogenetically as widespread as kinases. Prokaryotes have comparable numbers of kinases and NATs. In contrast, eukaryotes have on average 10 times more kinases than acetyltransferases. Taken together, this suggests that acetyltransferases originated close the root of the three domains of life.

Two structurally different domains are commonly employed for protein de-acetylation in all domains of life. Sir2 family enzymes, sirtuins (silent information regulators), are found in all three domains of life [59, 159, 185, 28] but are missing from genomes of many prokaryotes. In Eukarya and Archaea, these enzymes are NAD+ dependent and suggest an ancient link between chromatin structure and the metabolic state of the cell. Evidence for this comes from the conserved functional role of Sir2 even though it deacetylates different substrates in eukarya and archaea, namely, histones and Alba, respectively [17]. Rpd3-like de-acetylases have no similarity to Sir2 proteins. They are found in all eukaryotic genomes and have distant homologs in archaea and bacteria [63, 165, 185, 142, 127]. Another structurally different class is HD2-type histone deacetylases which appear to be present only in plants [127]. 

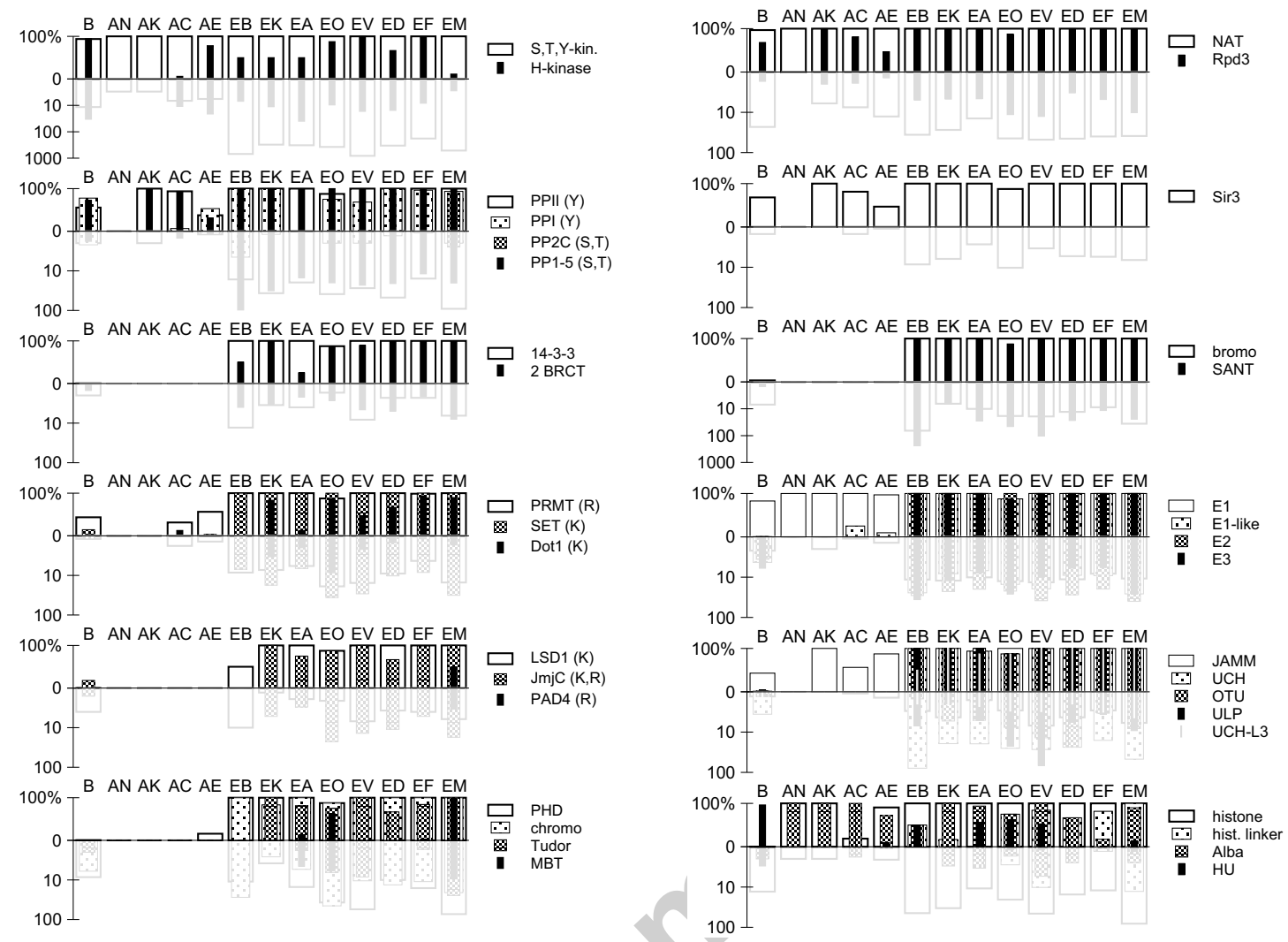

Figure 1: Phylogenetic distribution of functional domains involved in regulation of expression by chromatin. The upper panel of each individual graph shows the fraction of species per clade containing at least one protein with the functional domain specified to the right. The lower panel depicts the average over the absolute number of proteins for these species. While all domains of life utilize ChAPs to construct chromosomes, and modification writers (in particular phosphorylation, and acetylation) are present ubiquitously, we observe that modification readers are restricted to Eukarya.

Left panel from top to bottom: Enzymes for phosphorylation: Serine/Threonine/Tyrosine-kinases and Histidine-kinases. Enzymes for dephosphorylation: Tyrosine-phosphatases I and II, Serine/Threonine-phosphatases 1 to 5 and 2C. Reading of phosphorylation marks by 14-3-3 and tandem BRCT domains. Enzymes for methylation: protein arginine methyltransferases (PRMTs) and lysine methyltransferases SET and Dot1. De-methylation enzymes homologous to LSD 1 or JmjC and de-imination enzymes homologous to PAD4. Reading of methylation marks by PHD fingers, chromo, Tudor, and MTB domains. Right panel from top to bottom: Enzyme for acetylation: N-acetyltransferase (NAT). De-acetylation enzymes homolog to Sir3. Reading of acetylation marks (bromo domain) and unmodified side chains (SANT domain). Enzymes E1, E2 and E3 required for ubiquitination. Enzymes for de-ubiquitination: JAMM metalloprotease, Ubiquitin carboxyl-terminal hydrolases Otubain and Ubiquitin-like protein-specific proteases. Chromosomal architectural proteins (ChAPs): histones, linker histones, and homologs of Alba and HU. Phylogenetic clades: B - Eubacteria; Archaea: AN - Nanoarchaeota; AK - Korarchaeota; AC - Crenarchaeota; AE - Euryarchaeota; Eukarya: EB - basal eukaryots (e.g. Diplomonadida and Parabasilia); EK - Kinetoplastida (including Heterolobosea); EA - Alveolata (i.e. Ciliophora and Apicomplexa); EO - Chromista (i.e. Cryptophyta, Haptophyta, and Heterokonta) [191]; EV - Viridiplantae (incl. Chlorophyta); ED - Amoebozoa (e.g. Dictiostelium); EF - Fungi; EM - Metazoa. Note that the groups AN (Nanoarchaeota) and AK (Korarchaeota) each are represented only by one species.

We find that only a small fraction of prokaryotic species have at least one deacetylase, and that the numbers of these enzymes are very small in each species. In contrast, deacetylases are ubiquitous in Eukarya. It is interesting to note that there are at least twice as many acetyl-transferases than deacetylases per eukaryotic genome.

\section{3. (De-)Methylation}

Methylation can be manifold: Lysine residues can be mono-, di- or tri-methylated and arginine residues are subject to mono-methylation and symmetric or asymmetric dimethylation. All of these have been observed to play a role in chromatin modification [31]. Usually, the substrate for the enzymatic reaction is either S-adenosyl-L-methionin (AdoMet) or S-adenosyl- 
L-homocysteine (AdoHcy) [149]. However, the structural class of SAM-MTases does not include all AdoMet utilizing methyltransferases. Methylation of lysine can be carried out by SET (abbreviation for "Su(var), enhancer of zest, trithorax") [42] or Dot1 domain containing proteins. Less than one third of prokaryotic organisms have such proteins. There are no SET domain containing proteins in Archaea [185], consistent with our analysis. The only exception is a SET domain protein in Candidatus Methanoregula boonei which is homologous to bacterial Histone-lysine N-methyltransferases, probably acquired horizontally. We observe 3-15 times more Set than Dot1 domain proteins in Eukarya. Only Stramenopiles have at least one Dot1 domain in each genome. Several lysine residues of the archaeal ChAP Sul7d are subject to mono-methylation by an hitherto unknown enzyme [185, 107]. Furthermore, a SAMMTase in the archeon Pyrococcus horikoshii and its orthologs has been suggested to methylate rRNA and tRNA [161].

Methylation of Arginine is carried out by PRMTs (protein arginine-methyltransferase) [31, 16]. More than $95 \%$ of prokaryotes have at least one PRMT and all Eukaryots examined have at least two. The average number of paralogs per species is always lower than that of acetyl-transferases. Histone modifying PRMTs do not cluster within the family of PRMTs [16].

Eubacteria can have DNA, RNA as well as protein methyltransferases in their genomes. DNA and RNA methytransferases are involved in defense against foreign DNA or antibiotics [100]. Furthermore, protein methylation is found to play a role in sensory adaptation, where methylation is responsible for signal transduction and implementation of a short term memory [167].

Histone methylation has been thought irreversible until an amine oxidase reaction, carried out by LSD1 (lysine-specific demethylase), was proposed to cause de-methylation of mono- and di-methylated lysine residues $[155,173]$. At about the same time a hydroxylase with a JmjC domain was discovered able to de-methylate also tri-methylated substrates in a radical reaction $[175,173]$. The cell pays a high price for de-methylation as both reactions produce formaldehyde, a substance toxic to living cells because of oxidative stress. Deimination of methyl-arginine, carried out by PAD4 (peptidylarginine deiminase 4), also removes methylation marks but does not recover the arginine residue [180]. Although methylation is not irreversible, one expects massive reversal of methylation to be rare.

Figure 3 shows that there is a global correlation be- tween the number of modification and demodification enzymes in a given organism. With the exception of ubiquitination, "erasers" are substantially less frequent then "writers". Somewhat surprisingly, this difference is only moderate for methylation/demethylation.

\section{4. (De-)Ubiquitination}

Protein ubiquitination denotes the attachment of one or more ubiquitin molecules to a amino acid side chain, commonly a lysine. Ubiquitin is itself a small protein (76 AA in human) specific to eukaryotic cells. Similar polypeptides like SUMO ("small ubiquitin-like modifier") and RUB1 ("related-to-ubiquitin 1") can be attached to proteins in similar enzymatic reactions, termed sumoylation and rubylation [74]. The ubiquitination process requires a cascade of enzymatic reactions carried out by an activation enzyme (E1), a conjugation enzyme (E2), and sometimes a protein ligase (E3) [74]. The functional consequences of the latter modifications are just beginning to be determined. In general, polyubiquitination is a maker for protein degradation, while mono-ubiquitination plays a role in signaling and has be found in all major histone families and several histone variants $[179,183]$. Recent studies suggest that histone ubiquitination is a universal response to DNA damage and induces DNA damage repair [194].

Deubiquitination enzymes and other proteases, which detach ubiquitin-like proteins, belong to either metalloproteases or cystein proteases. At least seven protease linages with different evolutionary origin contain proteases with a cystein in the catalytic site [11]. Two contain the following protease domains relevant for de-ubiquitination: UCH and UCH-L3 (ubiquitin Cterminal hydrolases), OTU, and ULP (Ubiquitin-like protease). Other domains, e.g. Josephin, could not be found in the Superfamily database (see Section 10 for details).

Ubiquitination, de-ubiquitination and ubiquitin-like proteins have long thought to be completely absent in prokaryotes [45]. The discovery of small prokaryotic proteins with a $\beta$-grasp fold, as in ubiquitin, and $\mathrm{C}$ terminal activation by E1-like enzymes have changed this picture and suggest derivation of the ubiquitin conjugation system from the more ancient sulphur transfer pathway [75]. In addition, all canonical domains of life and viruses have members of both types of protease, cystein and metallo- proteases. This strengthens the evidence that ubiquitin-like proteins, and the corresponding conjugation system and proteases [123], trace back to the root of the phylogenetic tree.

The picture for the phylogenetic distribution of (de)ubiquitination enzymes is very similar to other marks. 
Both, relatives of some modifiers and demodifiers can be found in nearly all domains of life. The minimal set of necessary modifiers (E1 and E2) is present but still rare in archaea. The ratio between modifiers and demodifiers is different from other marks on the side of the modifier. The number of de-modifiers is sometimes higher then that of the modifiers, Fig. 3.

Summarizing this subsection, we detect a few general patterns. Most importantly, there is strong evidence that the evolutionary origin of chemical modifications leading to functional diversification of ChAPs predates the origin of eukaryotes [17]. These are common to (Eukarya+Archaea) but exclude Eubacteria [185]. Furthermore, the repertoire of modification writers is much more elaborate than that of modification erasers. In particular, not all Archaea appear to make use of erasers. Even in Eukarya, the use of writers and erasers is far from being balanced, Fig 3. This could be explained by the fact that modified ChAPs can also be removed by local replacement with unmodified proteins and subsequent degradation of the modified ones.

\section{The Evolution of Molecular Literacy: Modification Readers}

A major innovation that sets Eukarya apart from the other two domains is the invention of an elaborate signaling system based on chemical modification of ChAPs. In contrast to prokaryotes, chemical modification can be both placed and removed by specific enzymes and be recognized specifically. Reading is chemically and logically distinct from erasing modification: chemically, eraser enzymes recognize modifications with their active center; logically, a modification can be recognized only once by an eraser. In contrast, readers recognize the chemical modification and its context with an enzymatically inert domain; since the modification remains unchanged and can be read arbitrarily often.

\subsection{The Diversity of Reader Domains}

It is an intriguing observation that each type of histone modification is recognized by several distinct, apparently unrelated, protein domains $[40,166]$. We briefly summarize the major reader domains:

Bromodomains [118] read acetylation marks on lysine residues and are found in chromatin-associated proteins such as modifiers and remodellers. In addition, non-histone acetylation marks can be targeted. Proteins containing a double bromodomain bind two acetylation marks in a critical topological configuration, increasing specificity and affinity.

Methylation marks can be found on lysine and arginine residues. Lysine can be found in mono-, di- and trimethylated states in vivo. Several domains (e.g. chromodomains [48], chromo barrels [189], tudor, and MBT [1]) of the Royal superfamily can read lysine methylation marks and distinguish between the number of methyl groups. PHD-fingers also specifically recognize either methylated or unmethylated lysine residues. The key residues are often located at protein termini that can be inserted into the binding pocket. Methylation marks can also be placed on arginine. Mono- as well as symmetric and asymmetric di-methylation can be found.

Information on the corresponding readers is very limited, and no specific recognition proteins are known. WD40 repeats have evolved to detect many different signals, among them, histone methylation marks on lysine and arginine and histone phosphorylation marks. The $\mathrm{PhD}$ domains found in the archaeal genomes hit a hypothetical protein of Methanococcus maripaludis and the RecJ-like exonuclease of a few Pyrococcus species suggesting that these matches are almost certainly falsepositives.

14-3-3 proteins [117] are the most common readers of phosphoserine residues. Found in all eukaryotes, they are absent from all prokaryotes examined [51]. A tandem BRCT domain [157] can also recognize phosphorylation marks at the interface of domains. In budding yeast, readers of the latter type are, e.g. involved in cellcycle arrest in response to DNA damages marked by H2AX phosphorylation [69]. Only single BRCT domains can be found in eubacteria and archaea.

As for the modifying enzymes, we evaluated the phylogenetic distribution of reader domains in detail, Fig. 1. The most striking observation is that modification readers are almost exclusively found in eukaryotes. The few exceptions, mostly in bacteria, are found in a handful of species, who likely acquired them via horizontal gene transfer from an eukaryotic host. Among the major Eukaryotic clades, there is little variation in the distribution of reader domains, and there appears to be no or at most very little (sub)kingdom-specific innovation of relevant protein domains within the Eukarya. The number of genomic copies of particular domains, on the other hand, can vary by more than an order of magnitude between different clades, indicating clade specific patterns of diversification. The repertoire of phosphorylation readers (14-3-3 and 2BRCT domains) is almost two orders of magnitude smaller than the typical number of kinase domains, since kinases have functions beyond the regulation of chromatin. For methylation and acetylation, 


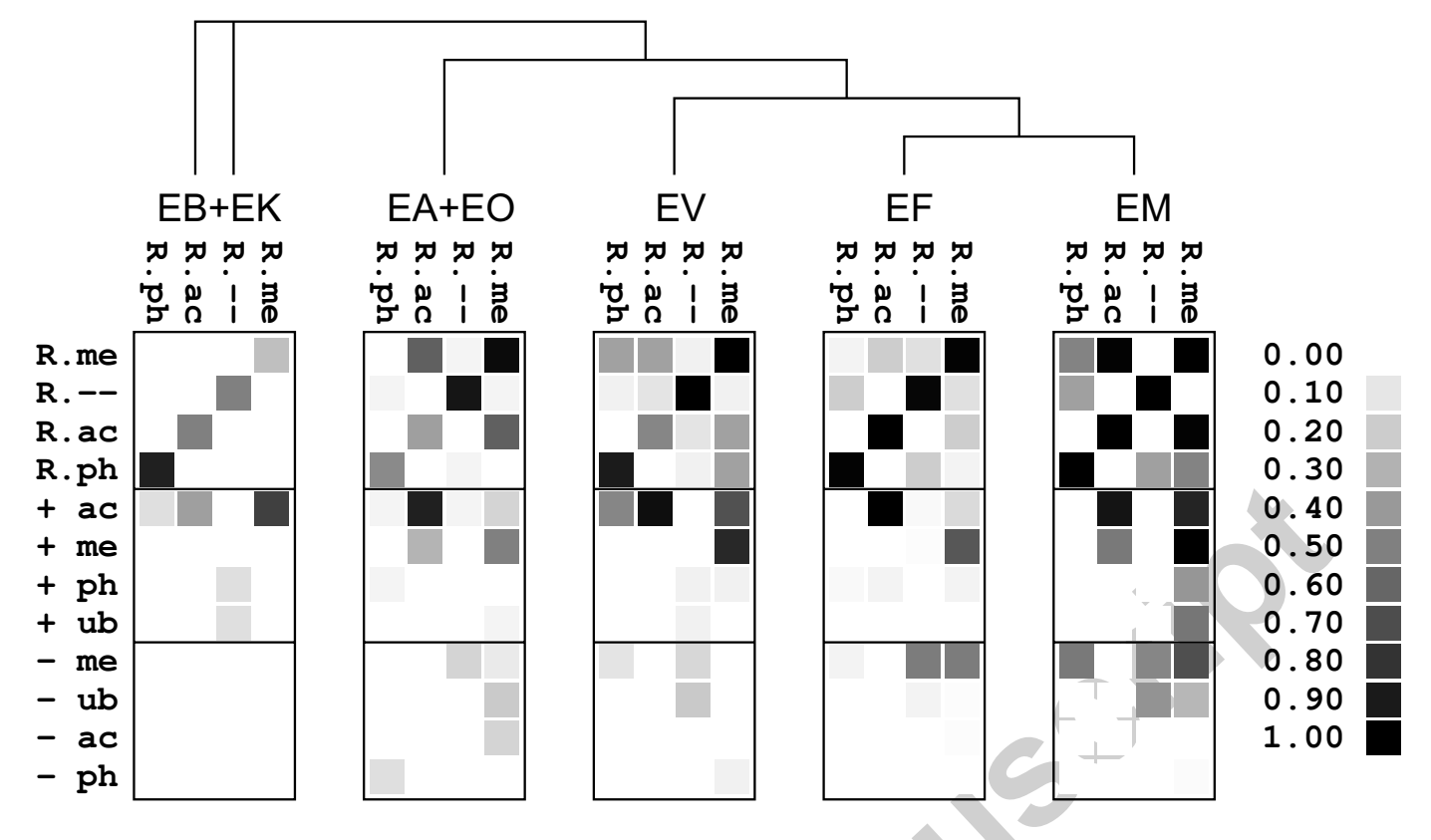

Figure 2: Phylogenetic distribution of protein domain co-occurring with reader domains. Reader domains are more often coupled with each other and with modification enzymes in crown-group Eukarya. Metazoa, in particular, have an extensive repertoire of such combinations that is highly conserved within the kingdom.

The grayscale values indicates the fraction of species in a clade that has at least one protein containing the domain combination specified by the left and top index of the matrices. 'R.me' - methylation reader; '+ me' - methylation enzyme; '- me' - de-methylation enzyme. Indices are analogous for ac - acetylation; ph - phosphorylation; ub - ubiquitination; R. - - reader of an unmodified side chain. EB+EK - basal eukaryots and kinetoplastids; EA+EO - Chromalveolata [108]; EV - Viridiplantae; EF - Fungi; EM - Metazoa;

the copy numbers of modifiers and readers are comparable.

Many eukaryotic chromatin-associated enzymes combine two or more distinct domains, Fig. 2. The combination of reader domains is likely to increase specificity and allows for recognition of more diverse modification patterns. Our data show that the use of reader domain combinations increases towards the crown-group Eukarya. While combinations of domains recognizing the same modification are present in most species, combinations of methylation and acetylation readers, for instance, are most frequent in Chromalveolata and Metazoa.

Previous studies have reported an enrichment of reader domains in chromatin modifying enzymes [40]. The analysis of the co-occurrence of reader and writer domains shows that acetylation is often coupled to reading of methylation marks. Animals in particular, appear to have an elaborate system of directing all four major types of modification, dependent on methylation and acetylation marks. Fungi, on the other hand, largely restrict themselves to writing methylation and acetylation marks depending on existing methylation and acetylation marks. Since marks may be set at nucleosomes adjacent to those that the enzymes read, the combination of reader and writer domains provides a means for the autonomous propagation of histone modification in response to histone-modification-dependent targetselection [53]. Combinations of readers and erasers are comparably rare. Only animals seem to use combinations of modification readers and demethylation domains in a systematic way.

Over all, we observe an increase in the diversity of combinations of reader writer and eraser domains in "higher" eukaryotes. It is also evident that combinations are more uniformly adopted in animals, and to a lesser extent in plants.

We emphasize that the analysis of domain cooccurrences does not exhaust the full complexity of the chromatin modification machinery. We anticipate protein complexes comprising several proteins with reader and writer domains permitting additional combinations. The domain co-occurrences nevertheless provide information on general evolutionary trends, and on function- 


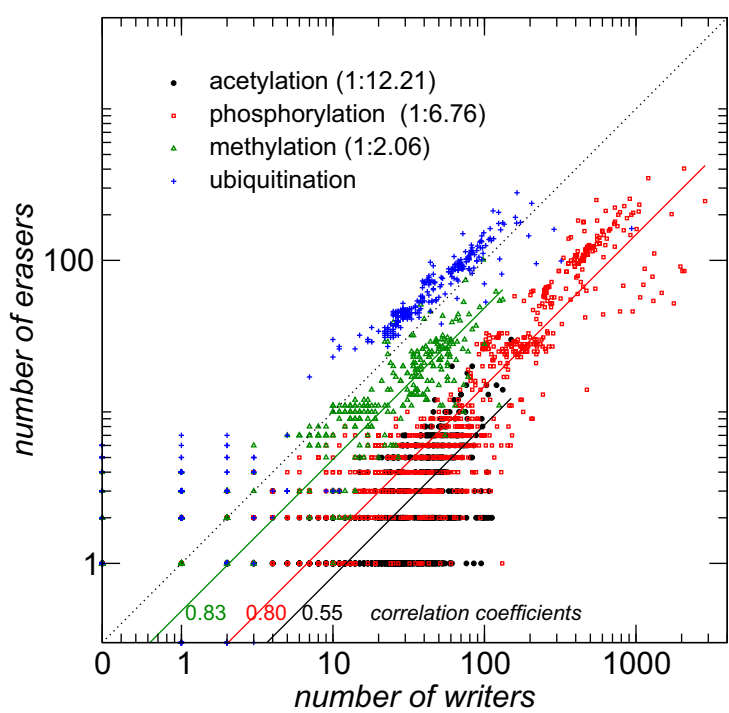

Figure 3: Correlation between the number of modification (writer) and demodification (eraser) enzymes. The effect is largest for acetylation with a ratio of more than 12 and phosporylation with a ratio of more than 6. For methylatiom the ratio is much smaller but still significant. In contrast, the bulk of species has slightly more ubiquitination erasers than writers. See text for more details.

alities that have preserved tight coupling over long evolutionary time scales.

\subsection{Guiding Chromatin-Modification to DNA Loci}

The function of chromatin in the regulation of transcription requires a connection between chromatin, the placement of variant nucleosomes, chemical modification, and the underlying sequence of genomic DNA. How this connection is established in practice, however, is by no means well understood at present. This is in part due to the diversity of documented mechanisms, which involve cis-acting DNA elements, nascent transcripts, and trans-acting RNAs. A particular location may be recognized in principle by any combination of DNA sequence information, chromatin state, and local genome activities (e.g. transcription). Corrsponding binding domain in chromatin modification complexes may give a hint on how the spatial positioning is achieved, Fig. 2. Nucleosome positioning further complicates the picture since it is influenced, but apparently not determined by DNA sequence features $[81,182,153]$.

Little is known about the detailed mechanisms that lead to the position-specific deposition of variant histones or other ChAPs. Histone chaperones and ATP- dependent remodeling complexes, which typically contain reader domains, are implicated in this process [82].

Similar to promoter elements or transcriptional enhancers, chromatin-opening elements [148], such as the HSFE involved in the regulation of $\beta$-globin expression [121], have been found that are involved in chromatin opening. Furthermore, interaction of sequence-specific DNA-binding transcription factors with chromatin modification enzymes has been proposed [33]. The middle panel of Fig. 4 shows that reader, writer, and eraser domains for specific modifications are frequently associated with DNA binding domains, demonstrating that there are extensive protein families that bring together DNA sequence information and chromatin modifications. In particular, histone demethylation appears to be firmly linked to DNA binding in all eukaryotes, implying that this process is at least in part directed by information residing in the DNA sequence. Only fungi and animals have a systematic repertoire of demethylases that are tied to modification readers. There is a general trends towards a tighter association between protein domains operating on chemical modifications and DNA binding domains in animals and to a lesser extent in the plants. Besides DNA sequence-dependent mechanisms and DNA-methylation dependent mechanisms $[164,56]$, process-dependent modifications have been described that store the recent local transcriptional history to chromatin states [73].

Several studies have demonstrated that siRNAs can promote DNA methylation as well as specific chromatin modifications in a number of lineages [30, 88, 98, 176]. Small RNAs are involved in targeting by direct interaction with the DNA or with nascent RNAs. While heterochromatin formation in $S$. pombe is guided by RNARNA recognition [29], plants appear to rely on direct RNA-DNA binding [129]. In ciliates, small scnRNAs are produced from the entire micronucleus, compared to the macronuclear DNA and degraded if the sequences match. After sexual reproduction, the retained scnRNAs target the DNA of the newly formed macronucleus to identify the "internal eliminated sequences" IES, trigger histone methylation, and eventually the excision of the corresponding DNA regions [93]. Plants have evolved an elaborate transcriptional machinery dedicated to eliciting sequence-specific, chromatin-based gene silencing [112].

Long, mRNA-like ncRNAs (mlncRNAs) play a crucial role in imprinting and other chromatin-level regulation that contribute to cell fate $[128,94]$. Recently mlncRNAs have been identified as crucial components in the polycomb/trithorax regulation system. The interaction of PcG and TrxG proteins with their target 

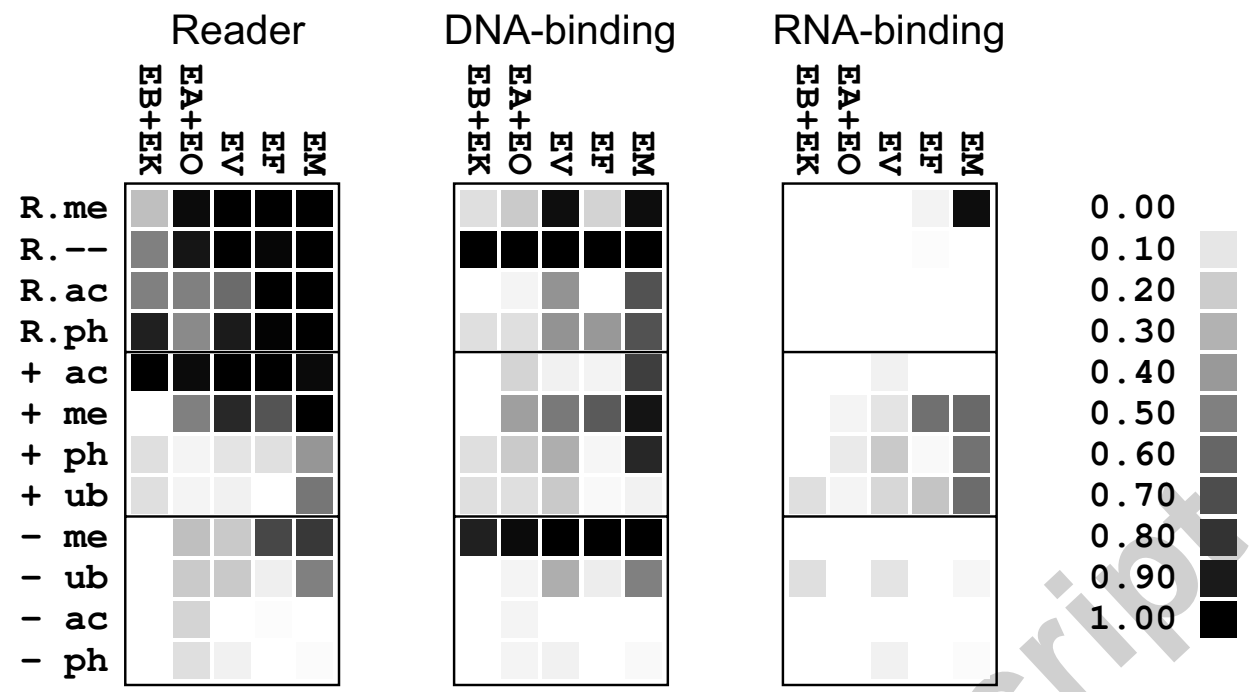

Figure 4: Evolutionary trends in domain combination of chromatin (de-)modifiers and three potential target selectors. L.h.s.: Combinations of reader domains are prevalent in all major Eukaryotic clades, while the coupling of reader and modifier domains increases towards the animals. In particular acetylation and methylation is coupled with reader domains. Middle: DNA binding domains are frequently coupled with modifier domains. In particular, demethylation appears to be governed by DNA information. Interestingly, a large class of proteins, which again increases towards animals, combines DNA binding with the reading of specific modifications. R.h.s.: The combination of RNA binding domains with chromatin modification shows a clear increase towards the animal clade. Abbreviations as in Fig. 2.

sites, the PRE/TREs (polycomb/trithorax responsive elements) can be achieved by several distinct mechanisms [71] that are also employed by other modification enzymes. The modifier complex may bind directly to a nascent complex, also proposed e.g. for the histone acetylase ASH-1 in flies [144]. Non-coding RNA may associate with their protein partners independent of the chromatin and guide them back to a target in cis or in trans recognized by RNA-DNA binding. This mode of action likely guides trans-actions such as the silencing of the HOXD cluster by HOTAIR [139]. Finally, nascent anti-sense transcripts might form anchors for ncRNAs-modifier complexes as suggested by the frequent bi-directional transcription from PRE/TREs. Recent data shows, that almost a quarter of the human mlncRNAs are physically associated with the repressive chromatin-modifying complex PRC2 [87], suggesting that mlncRNA play a crucial role in guiding chromatin modification .

In the r.h.s. panel of Fig. 4 we summarize cooccurrences of RNA binding domains with reader and modifier domains. There appears to be a strong increase in these combinations as we evolve towards the animal kingdom. In particular, the strong link between methylation readers and RNA binding is unique to an- imals. From these data it appears that chromatin modification in animals come to rely more heavily on specific RNA-binding than the other major groups of eukaryotes. This is consistent with the vast amount of mlncRNAs produced by animal genomes, which have not been reported, at least to this extent, for other clades. Plants, fungi and basal eukaryotes make extensive use of small RNAs in directing chromatin modifications. In this mode of action, the chromatin modifiers presumably interact with the RNP complexes of the RNAi machinery rather than directly with the small RNAs.

Fig. 4 appears to indicate an ancestral role for DNA binding domains and a prevalence of modifier-DNA interactions over modifier-RNA interactions. This could be the result of a strong annotation bias as nucleic acid binding domains are "by default" interpreted as DNA binders, and much less is known about RNA binding proteins in general. Zinc finger domains, here counted as DNA binding due to their function in transcription factors, for instance, are well known to also bind RNA $[26,68]$ and DNA/RNA duplexes [154]. The detailed distribution and relative importance of DNA- versus RNA-directed modifiers remains a question for future research. Irrespective of this outcome, the data show that there is a strong anchor that guides the chromatin 
modification machinery to sequence-specific loci. In particular (de)methylation is predominantly guided by nucleic acid sequence information.

\section{Epigenetic Inheritance of Chromatin Regulation}

An intriguing feature of chromatin is that it can transmit patterns of gene expression across cell divisions. This cellular memory is of crucial importance in the development of multicellular organisms and underlies cell differentiation. There is no single mechanism responsible for copying epigenetic information from one cell generation to the next. Instead, various combinations of modification and targeting mechanisms are combined to achieve a more or less faithful propagation of information. Rather few cellular transmission mechanisms are well understood, beyond the replication of DNA methylation by Dmnt1 [178]. Much less is known about mechanisms through which histone modifications are copied.

Histone modification does not prevent the transfer of parental histones to newly replicated DNA and thus are free to serve as a means of epigenetic inheritance. However, after replication, half of the nucleosomes must be assembled de novo, requiring mechanisms that "copy" chemical modifications forward, in order to establish faithful inheritance of the epigenetic marks [19]. The deposition of histone H3.3, for example, has been implicated in the inheritance of the active chromatin state [122]. Complex interactions of Polycomb and trithorax group proteins with several methylation and acetylation enzymes are necessary to maintain both inactive and active states and their boundaries $[150,151]$. Although critical details remain to be determined, it has become clear that some of the histone marks are regenerated after replication from partially transmitted information using the reader/modifier machinery [134].

For most chemical modifications it remains unknown whether they are epigenetically inherited. It is conceivable that the transcriptional status of chromatin, rather than the specific pattern of chemical modification, is transmitted to the next generation. We do know that chromatin-based information can be actively erased or reset upon replication $[116,138]$.

It remains to be determined to what extent epigenetic inheritance is tied to underlying DNA sequences. It has been established that histone modifications are not solely determined by the underlying sequence (in which case the "epigenetic" information would be a one-to-one mapping from the sequence information under the action of the modification enzymes). On the other hand, the epigenetic system is not a "free-floating" system, completely detached from DNA sequence information. Studies in fission yeast and other multicellular organisms suggest that effector complexes target nascent chromatin-bound non-coding RNAs and recruit chromatin-modifying complexes [3]. This has been suggested to contribute to the inheritance of chromatin states during the process of chromosome duplication [114].

Chromatin structure also plays a key role in DNA damage repair [49]. Nucleotide excision repair, a repair pathway conserved in animals and fungi, starts with histone modifications, in particular H2A monoubiquitinylation [194] that marks the damaged region. A complex remodelling cascade then enables repair by excision of the affected DNA segment and the reconstitution of the chromatin structure [193]. H3K9 modifications furthermore play a role in targeting class switch recombination in the Ig heavy chain gene [92].

The diversity and complexity of the mechanisms of epigenetic inheritance, as well as the apparently late evolutionary origin of several key components [77, $150]$, suggests that an elaborate heritable cellular memory was a late addition to the chromatin-based regulation machinery.

\section{A Hypothesis for the Evolution of Chromatin Regulation}

In this section we make use of our comparative, phylogenetic data, in combination with well known biochemical properties of DNA, RNA, and protein to propose an evolutionary series of events leading to complex forms of genetic regulation through chromatinmediated mechanisms. The scenario includes a number of testable hypotheses.

\subsection{Origins of DNA Genomes}

The early history of life presents us with many unsolved problems. Most researchers agree on the existence of an "RNA-Protein World" stage preceeding the divergence of Eubacteria, Archaea, and Eukarya, in which genetic information was stored in RNA [24, 61, $140,168,187]$. Two competing theories postulate either a last common ancestor (LUCA) of the three domains with a DNA genome $[13,109]$, or a LUCA with an RNA genome $[41,55,61,89,133]$. In the latter scenario, the transition to a DNA genomes occurred twice (once in Eubacteria and once in Archaea+Eukarya) [54] or even thrice [55], possibly mediated by viral entities. The literature agrees, however, that complex cellular machineries were present in the ancestral RNA genomes 
before they were replaced by modern DNA-based information storage, and that this substitution was driven by the advantages of a chemically and thermodynamically more stable genomic material [24].

A late and multiple transfer of a large amount of genetic information from RNA to DNA implies that the regulation of protein expression must have been predominantly translational in the ancestral RNA state. The logical alternative is specific differential regulation of the replication of specific RNA fragments ${ }^{2}$ [168]. It is unlikely that such a mechanism will have been shifted over to a new DNA chromosome with the same RNA and protein factors in place. This is because RNAbinding proteins do not necessarily bind DNA, and even if they do, there is no chemical reason for them to recognize the same nucleotide sequences.

We propose that the transition from an RNA to a DNA genome was most likely from an ancestral state in which the protein-production was regulated predominantly at the level of translation. A generic transcription machinery, perhaps of viral origin, without complex fine-grained regulation would be capable of reproducing an RNA complement similar to that of the former RNA/protein world ancestor, so that the ancestral post-transcriptional mechanism could continue to function.

Gene-specific transcriptional regulation, we argue, must have been a later innovation. Hence this generates the hypothesis that specific transcription factors should have arisen towards the distal branches of the tree of life. This hypothesis is supported by the observation that specific regulation by transcription factors is evolutionary plastic, with global transcription factors poorly conserved across phylogeny [102]

\subsection{ChAPs as Early Transcriptional Regulators}

ChAPs probably arose together with the DNA genome, presumably for one or more of the following reason:

1. In a scenario where DNA was imported from viruses, it may have been necessary to distinguish cellular from foreign DNA.

2. A relatively large DNA genome may have required some architectural stabilization to prevent agglutination.

3. ChAPs may have evolved as a simple mechanism for separating a transcriptionally active state from a

\footnotetext{
${ }^{2}$ Models for "transcription" in the RNA world typically envision this as a replication-like process mediated by an ancestor or relative of the ribosome.
}

replication phase. The ability to "decide" whether to replicate or not could provide substantial advantage in nutrient poor and highly variable environments.

4. The stable DNA genome may have enabled the formation of durable "spores" capable of surviving harsh temporary conditions.

Initially, only the global transcriptional activity would be regulated, presumable by means of the concentration of ChAPs. Differential protein expression, on the other hand, was still organized at the RNA-protein level - translational - just as in the ancestral RNA/protein organism. This leads us to hypothesize that ChAPs along with many other DNA binding proteins, such as transcription factors, originated from ancestral RNA binding domains. Support for this hypothesis comes from the fact that similar folds can bind both classes of nucleic acids $[26,65,190]$. The diversity of ChAPs in the different domains is consistent with independent origins of DNA genomes in Eubacteria and the Archaea/Eukarya lineage.

Paralogous ChAPs with somewhat different sequence preferences have arisen by gene duplication, providing a potential means of distinguishing chromosomal regions in which transcriptional activity could be regulated differentially through modulation of the concentration and relative abundance of paralogous ChAPs. Recall that such mechanism are still used in e.g. distinguishing exponential and stationary growth phases in Eubacteria and Archaea. Most plausibly, at this stage, the regulatory effect would be exerted directly by the physicochemical properties of the ChAPs.

The earliest forms of transcriptional regulation were thus generalized and repressive, and entirely dependent upon a small collection of ChAPs. This defined a handful of chromatin states on a single genome that mapped onto multiple clearly distinct, phenotypic states. Sequence-specific transcription factors would have evolved to allow activating exceptions to the general repressed states refining/backing up the specific regulation of expression implemented on the level of translation. Eventually, this leads to the current solution, in which the expression of a typical protein is subject to both transcriptional and post-transcriptional regulation $[10,78]$.

Chemical modification of ChAPs offers a more economical alternative to the use of paralogs, and could have co-opted enzymes that originally modified other proteins in a host-defense or signaling context. Chemical modifications provide a faster means of responding to external signals than the expression of ChAP par- 
alogs, and reduces the metabolic burden imposed by ChAP synthesis. The combination of a small set of non-specific DNA-binding ChAPs, and several types of modifications set by specifically targeted modification enzymes, allows for a fine-grained definition of chromatin states.

The imbalance in the prevalence of modification "writers" over modification "erasers" hints at a later addition of general demodification activities. There are at least three interpretations for this observation: (1) Harmful substances are produced during the demodification reaction (e.g. demethylation) causing selection against the massive use of such a reaction pathway. (2) a very general de-modification mechanism is already in place since replication and ChAP turnover thins out modified ChAPs through re-synthesis of unmodified ChAPs. The controlled degradation of modified ChAPs could therefore quickly return the chromatin to the unmodified state, rendering specific demodification an unnecessary step. Once multiple modifications are written on the same nucleosome, the degradation pathway becomes impractical. (3) A cascade of specific, effectively irreversible modifications may have been evolutionarily advantageous as a means of implementing asymmetric differentiation of cell states, thereby setting the stage for organismal development. Unfortunately, we cannot entirely rule out that the under-representation of erasers is caused by a bias in the protein domain data.

\subsection{ChAPs as a Memory Device}

The invention of reader domains in the ancestral Eukarya turned chromatin into a cellular memory device. Chemical marks written onto the ChAPs in the cellular past can now be interpreted and modified in a contextsensitive fashion by proteins that combine reader domains and effector domains. It allows the cell to keep a record of former states - in particular, of the past transcriptional activity in any given genomic region. A major advantage of such a setup is that the transcriptional programs no longer needs to be activated or terminated using direct feedback e.g. through measurements of metabolites or environmental factors.

Interestingly, we found that the reader/effector system is quite variable among the various Eukaryotic lineages. Many of the protein families that implement specific combinations of reader and effector domains are exclusive to specific clades, with much of the combinatorial complexity of readers and effectors explored throughout the course of evolution.

Neither the advent of a reader domain nor the innovation of reader/effector combinations are particularly unlikely events. Fusion proteins and recombinations of protein domains are abundant throughout protein evolution. It is perhaps more surprising that Archaea and Eubacteria do not seem to have evolved a ChAP-associated memory system. We speculate that this is not for lack of opportunity but rather for the lack of immediate advantage or access.

The combination of reader domains with writer and eraser domains enables a network of histone modification in response to histone-modification-dependent target-selection [53] and promotes an autonomous dynamical system resulting in epigenetic information cascades superimposed onto and uninfluenced by the underlying DNA $[18,52,67,152]$. The potential detachment of the chromatin modification network from underlying DNA is prone to conflict with the adaptive requirements of transcriptional regulation. We hypothesize that this semi-independence could be a source of pathology, such as in cancer. It is no surprise, therefore, that transcription and histone modification are tightly linked. A variety of distinct mechanisms, from DNA binding enhancers [91] to the employment of small and large ncRNAs have evolved to anchor the modification activities of the chromatin to the underlying DNA sequence.

\subsection{Epigenetics}

Epigenetic inheritance is the most recent control layer in the chromatin regulation system. In fact, it does not consist of a single coherent mechanism but a collection of rather elaborate (and somewhat mysterious at this point) "tricks" to propagate selective information stored in the chromatin memory to daughter cells. To the extent that epigenetic inheritance is understood at all, it re-utilizes diverse components of the chromatinregulation machinery to regenerate part of the cell stateinformation following stochastic assortment of the histone complexes to daughter cells. Very little is known to what extent the detailed mechanism are lineagespecific. Organismal development depends on this process to propagate epigenetic states across cell divisions and to implement a program of differentiation steps that are effectively irreversible.

\section{Chromatin Computation}

\subsection{Gene Regulation as Computation}

Gene regulation can be thought of as a form of computation. So far, this point has been made most explicit in the case of cis-regulatory networks [23, 76, 96]. Cisregulatory modules (CRMs) are abstracted as Boolean 
Functions that combine the input - a pattern of currently present transcription factors - by means of conjunction, disjunction and complementation (AND, OR, and NOT gates). Complex circuits are formed since both "down-stream effectors" as well as transcription factors themselves are regulated by such CRMs. This cis-regulatory model of genomic computation represents one of the essential computational modalities of the cell, and has been shown to play a crucial role in development.

Here we suggest that the functional interpretation of our phylogenetic findings and evolutionary hypothesis is that chromatin regulation adds a computational layer that, in Eukarya, is qualitatively different and potentially more powerful than the CRM networks. As we shall see, the difference is the explicit and extensive memory implemented in the ChAP modifications. In order to proceed, we need to introduce the idea of "computation" generally and more formally, which we can view essentially as statement about constraints on input-output functions. Stated this way, we shall see that CRMs are but one class of important mechanisms for information processing. We start from a set of basic or atomic states $S$ and a transition operation $\rightarrow$ operating on these states, which is simply a relation $\rightarrow \subseteq S \times S$ on the set of states that tells us which transitions are allowed. Formally, a computation is then simply a sequence $\left(s_{1}, s_{2}, \ldots, s_{n}\right)$ of states $s_{i} \in S$ that are related by $s_{i} \rightarrow s_{i+1}$. The set of all computations w.r.t. $\rightarrow$ will be denoted by $M$. For computations to be effective they need to both come to an end (or halt $H$ ) and produce a result that is available or readable by some other components of a system, $R$. Hence we need to introduce a stop predicate $H: M \rightarrow\{0,1\}$ and consider only the computations $M_{H}$ that halt. For these we further require an "output mapping" $\rho$ that takes the results of a complete computation and makes them available for evaluations: $\rho: M_{H} \rightarrow R$. A computing system $\Gamma$ is then simply defined in terms of the quadruple: $\Gamma=(S, \rightarrow, R, \rho)$.

This formal specification is still too abstract for our purposes. Let us therefore think of each state $s \in S$ as a particular realization of a biological structure that is composed of elementary objects that can be manipulated individually during the state transitions. The abstract transitions $\rightarrow$ then become concrete rewriting operations on collections of these elementary objects/data structures. In the simplest case, $s$ is string over some alphabet $\mathcal{A}$. In this particular implementation, computer science defines a so-called Chomsky hierarchy [32] that establishes a correspondence between the form of the rewrite operations, computational power, and the structure of the transition rules that implement a computa- tion.

\subsection{Memory Capacity}

CRMs are naturally interpreted in terms of finite state machines. Each state $s$ corresponds to a particular concentration profile of a few hundred to a few thousand regulators, mostly transcription factors, signalling proteins, and microRNAs. Each of these regulators encodes in its concentrations, a small number of states that are distinguishable at the transition level, and hence contributes a few bits to the overall storage capacity of the system. A crude upper bound can be obtained from the number of regulators, $n_{\text {reg }}$, and their number of copies $N$ in the cell: Even assuming that half the human genes are regulators, $n_{\text {reg }}=10^{4}$, with 1000 copies each, there are no more than $10^{5}$ bit of information in a CRM network.

In chromatin computation, each state $s \in S$ is the particular arrangement of all the specifically modified ChAPs on the DNA. Since little is known about the feedback between rearrangements of $\mathrm{ChAP} /$ nucleosome positioning (chromatin remodeling) and chemical modifications, we simplify this picture by neglecting the details of nucleosome positioning. In this approximation, a state $s \in S$ is then a particular pattern of ChAP modifications and variants in the linear array of nucleosomes. This arrangement is similar to the linear memory organization of a digital computer. A nucleosome then corresponds to a particular page of memory. The organization of each page deviates in details from a simple string because different residues can sustain different numbers of modifications of different types.

In the absence of readers, chemically stored information is not persistent in that it cannot be utilized without changing it. Demethylation, therefore, is fundamentally different from reading a particular methylation pattern. With the advent of reader domains, however, the same piece of information can be accessed repeatedly and in different contexts. As a consequence, eukaryotic chromatin can store information on a much longer time-scale than CRMs.

Eukaryotic nucleosomes have a sizable memory capacity owing to the different combinations of methylation, acetylation, phosphorylation, and ubiquitination that can be present or absent at multiple residues in each of several ChAPs forming a single nucleosome. Based on published histone modification maps, yeast can store up to about 70 bits of information in a single nucleosome, in human the capacity can be estimated as $\sim 200$ bits (see Methods section for details). Note that this value is comparable to a memory capacity of not more than 400 bits provided by the approximately 200nt of DNA in the the sphere of influence of a nucleosome. 


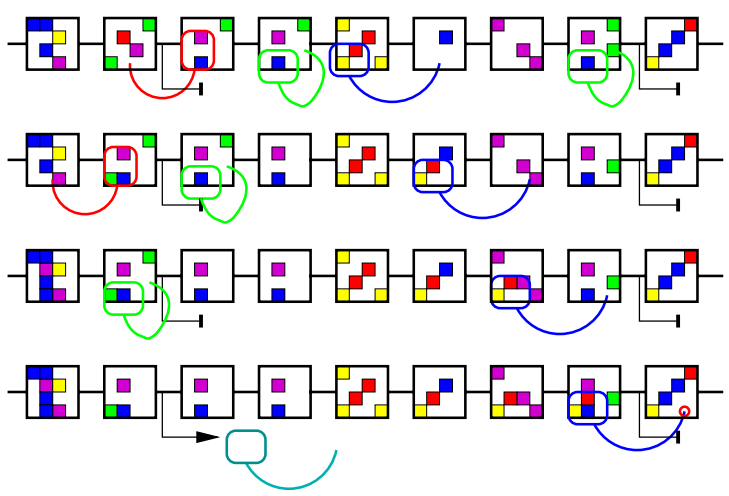

Figure 5: Computational Model of Chromatin. Memory is organized in a linear sequence of pages (nucleosome) and all state changes are restricted in a Cellular-Automaton-like fashion to local neighborhoods. State transitions are determined by rewrite rules (Reader/Modifier complexes) that recognize (part of) the information stored on a particular memory page (nucleosome) and change (some of) the content of the same or an adjacent memory page. The set of available rules also depends on the state of particular local memory pages (indicated by the 'transcription start site symbols'), corresponding the transcriptional activity of particular Reader/Modifier complexes.

Since the number of accessible states is still finite, we could formally map chromatin computation to a finite state machine, albeit with a state space that is 4-6 orders of magnitude larger than that of CRMs. A more natural interpretation is that chromatin computation implements a context-sensitive mechanism with a more modest state space.

We can extend this computational interpretation by recognizing that the transitions in eukaryotic chromatin computation are typically local and massively parallel at the same time. Each reader/writer recognizes a subpattern of just a few bits on each memory page and writes/modifies a few on the same or an adjacent nucleosome. The chromatin modification machinery is thus reminiscent of a vector or even parallel computer, Fig. 5. Each of the memory pages (nucleosomes) is subject to the transitions caused by a particular combination of reader and modifier enzymes active at any given time. If the chemical activity is uniform across the genome, the system behaves much like a vector processor. In conjunction with region specific targeting systems, different modifications processed can be active concurrently in different regions of the genome/memory, so that chromatin computation becomes a kind of imperfectlysynchronous, parallel computing.

\subsection{Biochemical support of chromatin computation}

Transcriptional regulation, in this computational paradigm, is the result of associating a computational process to specific biochemical effectors. Here we discuss the detailed biochemical micro-structure of our apparatus in terms of symbolic operations. A selec-

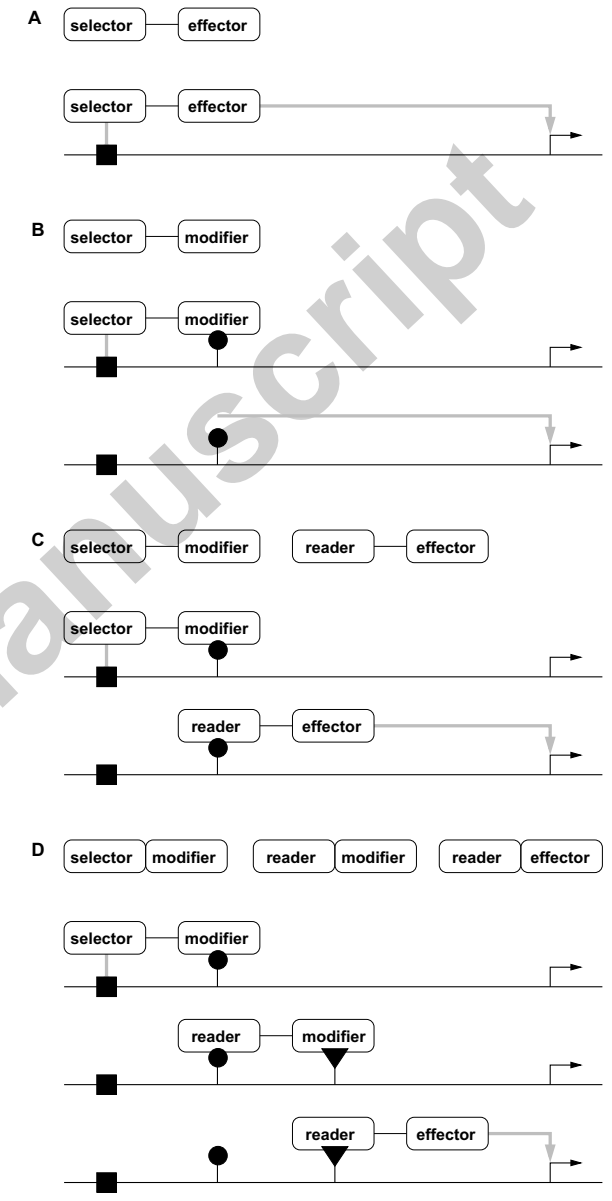

Figure 6: Conceptual innovation in chromatin regulation. (A) Direct effects on transcription are carried out by specific or unspecific selector-effector pairs, such as transcription factors or variants of chromosmal architectural proteins (ChAPs). (B) Only Archaea and Eukarya are known to chemically modifiy their ChAPs. Initially, it is this modification itself that effects the binding affinity of the protein and therefore regulation. (C) Modification readers are unique to Eukarya. Now a signal can be set by a selector-modifier. This persistent signal is then interpreted by a reader-effector combination. The modification thus becomes a signal in an information theoretic sense and provides a means for separating the decision making process from the execution of the decision. (D) The coupling of reader and modifier allows signal propagation along the DNA and provides the physical basis for signal inheritance and complex computations. 
tor guides an effector to a particular genomic position and induces or prevents transcription at a given locus. In CRMs, this selector is the DNA binding domain of a transcription factor binding to specific DNA locus, Fig. 6A. Here, the presence of the transcription factor is required to exert the desired effect. This appears to be the dominant mode of regulation in Eubacteria. Using a programming analogy, it corresponds to an if then statement. Logically related is the use of a selectormodifier combination. In the case of the deposition of variant nucleosomes and the writing or histone modifications in Archaea, the modification itself physically influences transcription; the effect persists as long as the modification is present, Fig. 6B. The important distinction from CRMs is that in the latter case modifications have only transient memory for the action of a modifier. From a programming point of view, modifications may be seen as while statements. The combination of effectors with modification readers expands the use of modifications as memory to include interpretation of the stored information in different ways, Fig. 6C. Chromatin in higher eukaryotes appears to be utilized in this way because most modification marks do not have unambiguous interpretations, but their function depends on the cellular context. Reader-modifier combinations, finally, allow the implementation of complex programs without the need to produce an immediate effect on transcription at all, Fig. 6D.

CRMs and chromatin differ in a second important property. Most of the states of CRMs are subject to selective constraints because the expression of regulatory gene products, almost by definition, has an impact on the production of downstream target genes. Transitions between regulatory states therefore can be expected to have direct physiological consequences. Changes in the regulatory program are typically immediately visible to the forces of natural selection, implying rather stringent constraints on the computational programs that "can be run" on a CRM.

In contrast, chromatin modifications have rather indirect, aggregate effects on transcriptional activity, such that a large fraction of its memory capacity can be utilized without direct physiological effect of the chemical modifications themselves. Hence it is feasible to implement rather elaborate computations in chromatin which are selectively (nearly) neutral (except for the resource consumption of the modification chemistry itself).

\section{Concluding Remarks}

We have surveyed the phylogenetic distribution of mechanisms of chromatin modification and regulation.
Chromosomal architectural proteins (ChAPs) and chromatin are present throughout extant organisms and have a demonstrable impact on gene expression. Multiple alternative ChAPs and paralogous variants of ChAPs influence chromatin structure. The mode and extent of chromatin regulation is quite different in Eubacteria, Archaea, and Eukaryotes. In contrast to Eubacteria, both Archaea and Eukarya regularly utilize chemical modification of ChAPs, which in Archaea is largely restricted to setting modifications with immediate biophysical impact. In contrast, Eukarya possess an elaborate system for managing chemical modifications comprising proteins that can write, read, and erase in a highly specific way post-translational modification such as phosphorylation, methylation, acetylation and ubiquitination. A phylogenetic analysis of domain-occurrences indicates increasingly complex interactions between reading and modifying (writer and eraser) domains in the crowngroup Eukaryotes.

We view the regulation of gene expression through chromatin regulation as a parallel information processing system that is evolutionarily prior to the transcription factor based templating machinery stressed in cisregulatory modules. It is only with the appearance of the Eukarya, that chromatin regulation transitions from a write-delete system to write-delete-read system. This suggests to us that with the Eukarya we move from a crude low-dimensional ancestral finite-state computing device, to a highly tuned parallel computing architecture with a significantly expanded memory capacity.

The regulatory complexity that can be achieved (in the form of CRMs) through DNA-binding proteins is somewhat limited by their binding properties [110]. As a consequence, small non-coding RNAs have recently been proposed to serve a better function in specific and selective DNA recognition with the additional benefit of genome compactness of ncRNAs and evolvability of the interaction networks $[110,111,135]$. We know that many of these RNAs appear to be involved as selectors for action on chromatin. We suggest that chromatin regulation could provide significant increases in both the resolution of cellular information processing and contribute to fault tolerance by providing a redundant layer above CRM mediated regulation.

With the appearance of the Eukarya we see the emergence of complex forms of multicellular life, and it is tempting to speculate that this was correlated with the expansion of computational power. We know that differentiated multicellular organisms rely on epigenetic processes to retain cell states, and that these epigenetic marks are often carried by chromatin. Here we suggest that epigenetics in the form of chromatin modifying sys- 
tems adds a layer of context-sensitivity and thereby regulatory flexibility during the developmental process.

Because chromatin regulation presents an additional, complementary, regulatory level to that provided by cisregulation, the regulatory layers might diverge when they behave in ways that are fitness neutral. Hence extensive write-read-write operations at the chromatin layer that have a minimal impact at the level of cisregulation (and hence translation) will not be inhibited by anything other than the potential cost of frequent chemical modification. Hence there is the possibility for extensive "free-wheeling" at the chromatin level, generating significant variation that could come to play an important adaptive role (both negative and positive) with a change in cellular context. The most obvious example is cancer as a chromatin disease [50]. Since DNA repair mechanisms appear to be programmed by chromatin, perhaps the increasing chromosomal aberrations in many cancers are not just incidental DNA damage, but the downstream effect of free-running chromatin dynamics loosely coupled to immediate selective consequences.

In the germline, the nearly complete erasure of chromatin marks could be a "precaution" against the accumulations of computational errors at the chromatin level: the propagation of epigenetic information is limited by Eigen's error-threshold [46, 47]. This theory implies that extremely high accuracies on the order of a single copying error per replication are required for the long-term information maintenance. With the possible exception of DNA methylation, mechanisms of epigenetic inheritance appear to be much noisier, which could explain why they are restricted to somatic cells. With largely unmarked chromatin as a starting point, early embryonic development could hardly be governed by chromatin. Instead, CRMs have been tremendously successful in regulating these processes. The transcriptional activity in these early stages also leads to an accumulation of chromatin marks, which soon take control of the cell fate.

These findings, taken together, provide evidence for the evolution of complex, combinatorial forms of information regulation, starting from simple structural precursors. Moreover, multiple regulatory mechanisms act in parallel, and in all likelihood, redundantly. These facts attest to the inability of complex regulatory systems to suppress all sources of uncertainty.

\section{Acknowledgements}

This work was supported in part by the VW Foundation and the Pathfinder initiative on Complexity through the projects EDEN (043251) and SYNLET (043312). In order to keep intact our chances for future federal funding we refrained from using the title "How to get histoned" for this contribution.

\section{Methods}

\subsection{Phylogenetic Distribution of Protein Domains}

Domain annotation for 751 species (1099 strains and/or different genomes) were extracted from the genome assignment data of the Superfamily database [186] (version 14-Jun-2009) in flat file format.

Domains relevant for chromatin regulation were derived from known proteins involved in chromatin regulation taken from the literature, and their domain annotation as given by scop [4] and Superfamily.

Phylogeny. Species (with one to several strains each) were assigned to the following clades, B - Eubacteria; A - Archaea; E - Eukarya; AN - Nanoarchaeota; $\mathrm{AK}$ - Korarchaeota; AC - Crenarchaeota; AE - Euryarchaeota; EB - basal eukaryots (e.g. Diplomonadida and Parabasilia); EK - Kinetoplastida (incl. Heterolobosea); EA - Alveolata (i.e. Ciliophora and Apicomplexa); EO - Chromista (i.e. Cryptophyta, Haptophyta, and Heterokonta) [191]; EV - Viridiplantae (incl. Chlorophyta); ED - Amoebozoa (e.g. Dictiostelium); EF - Fungi; EM - Metazoa, based on the deep phylogeny presented in [77] for critical splits and the "Tree of Life" or NCBI taxonomy otherwise. Monophyletic Chromalveolata $(\mathrm{EA}+\mathrm{EO})$ with Chromista and Alveolata as sister groups [108] were assumed here.

Domain distribution. For each domain, gene counts were maximized over all strains for each species and averaged over all species, with at least one gene, for each clade. This reflects the average abundance of a domain $X$ within a clade. Furthermore, we computed the fraction of species within a specific clade that has at least one gene with domain $X$, indicating the prevalence of domain $X$ within a clade.

Computational Tools. The domain abundance and prevalence values for single domains or domain combinations are computed with a sequence of awk and sort commands together with simple perl scripts using the Superfamily data described above and phylogenic information as input. 


\subsection{Information on DNA versus Nucleosomes}

The amount of information that can be stored by a nucleosome was calculate based on the number of different sites and types of modifications under the assumption that all sites on the eight histones can be modified independently. A comprehensive list of possible modifications for mammalian histones was collected from the literature:

Modifications of histone H3: H3R2me [22, 15, 171], H3R2cit [37], H3T3ph [22], H3K4me [22, 60], H3K4ac [60], H3K4bio [90], H3R8me [22], H3R8cit [37], H3K9ac [22, 60], H3K9bio [90], H3K9me [22, 60],

\begin{tabular}{|l|l|l|}
\hline group & domain & superfamily IDs \\
\hline ChAP & HU & 47730 \\
& Alba & 82704 \\
& histone & 47114,47129 \\
& linker histone & 46827 \\
\hline Mub & E1 & 46935,69572 \\
& E2 & 54496 \\
& E3 & 56205 \\
\hline Dub & UCH & 82568 \\
& UCH-L3 & 54050 \\
& ULP & 54054 \\
& OTU & 110773 \\
& JAMM & 102712 \\
\hline Mph & H-kinase & 55884 \\
& STY-kinase & 88854 \\
\hline Dph & PPI & 52788 \\
& PPII & 52799 \\
& PP1-5 & 56310 \\
& PP2C-like & 81601 \\
\hline Rph & 14-3-3 & 48446 \\
& BRCT & 52113 \\
\hline Mac & NAT & 55730 \\
& Rpd3 & 52773 \\
\hline Dac & Sir2 & 63984 \\
\hline Rac & bromo & 47370 \\
\hline R- & SANT & 46739 \\
\hline Mme & SET & 82200 \\
& PRMT & 53351 \\
& Dot1 & 89746 \\
\hline Dme & JmjC & 82194 \\
& LSD1 & 140222 \\
& PAD4 & 110107 \\
\hline Rme & chromo & 54165 \\
& MBT & 89299 \\
& PHD & 57911 \\
& Tudor & 63749 \\
\hline & & \\
& SAD & \\
\hline
\end{tabular}

H3S10ph [22], H3T11ph [22], H3K14ac [22, 60], H3R17me [22], H3R17cit [37], H3K18me1 [60], H3K18ac [22, 60], H3K18bio [90], H3K23me1 [60], H3K23ac [22, 60], H3R26me [22], H3R26cit [37], H3K27me [22, 60], H3K27ac [22, 60], H3S28ph [22], H3P30iso [120], H3K36me [22, 60], H3K36ac [22, 60], H3P38iso [120], H3K56me [60], H3K56ac [22, 113, 60], H3K79me [22, 113, 60], H3K79ac [60], H3K115ac [192], H3T118ph [57], H3K122me [57], H3K122ac [192].

Modifications of histone H4: H4S1ph [22], H4R3me [22], H4R3cit [37], H4K5ac [22], H4K8ac [22], H4K8bio [90], H4K12ac [22], H4K12me1 [192], H4K12bio [90], H4K16ac [22], H4K20me [22, 60], H4K20ac [192], H4K31me [14, 60], H4K31ac [60], H4S47ph [57], H4R55me [14], H4K59me [57], H4H75ph [21], H4K77me [14], H4K77ac [192], H4K79ac [192], H4K91ac [113], H4R92me [57].

Modifications of histone H2A: H2AS1ph [22], H2AK5ac [22], H2AK9ac [22, 12], H2AK9bio [90], H2AK13ac [22, 12], H2AK13bio [90], H2AK15ac [12], H2AK36ac [12], H2AK99me [57], H2AK119ub [22], H2AT120ph [22], H2AK125ac [12], H2AK127ac [12], H2Ak129bio [90]

Modifications of H2B: H2BK5ac [22], H2BK11ac [12], H2BK12ac [22], H2BS14ph [22], H2BK15ac [22], H2BK16ac [12], H2BK20ac [22], H2BK23me [192], Н2BK23ac [12], Н2BK24ac [12], Н2BK43me [192], H2BK47me [14], H2BK57me [14], H2BK85ac [192], H2BR99me [192], H2BK108me [14], H2BK108ac [192], H2BK116ac [12], H2BK120ub [22], H2BK120ac [192]. Based on the sparsity of functional information on non-enzymatic histone biotinylation [70], we excluded these marks from further calculations.

The information content (in bits) is calculated by summing over the logarithm (to the base 2) of different states per site for a histone octamer (i.e. $2 \times \mathrm{H} 3,2 \times \mathrm{H} 4$, $2 \times \mathrm{H} 2 \mathrm{~A}, 2 \times \mathrm{H} 2 \mathrm{~B})$. Notice that the unmodified state is a state and that lysine and arginine methylations each contribute three states (mono-, di-, or tri-methylation and mono-methylation, symmetric or asymmetric dimethylation, respectively.) The total information content is 205.84 bits $\left(I_{H 3}=38.65, I_{H 4}=25.87, I_{H 2 A}=\right.$ 14.17, $\left.I_{H 2 B}=24.23\right)$.

The storage capacity of DNA can be estimated as $I_{D N A}=2 L$ since each position contributes $\log _{2} 4=2$ bits for the four states A, C, G, or T. Assuming one 
nucleosome per $200 \mathrm{nts}$, the DNA has an information content of 400 bits per nucleosome position. Epigentic information thus may constitute up to $1 / 3$ of the total information stored on a chromosome.

\section{References}

[1] M. A. Adams-Cioaba and J. Min. Structure and function of histone methylation binding proteins. Biochem Cell Biol., 87:93$105,2009$.

[2] K. Ahmad and S. Henikoff. The histone variant H3.3 marks active chromatin by replication-independent nucleosome assembly. Mol Cell, 9:1191-1200, 2002.

[3] P. P. Amaral and J. S. Mattick. Noncoding RNA in development. Mamm Genome, 19:454-492, 2008.

[4] A. Andreeva, D. Howorth, J.-M. Chandonia, S. E. Brenner, T. J. P. Hubbard, C. Chothia, and A. G. Murzin. Data growth and its impact on the SCOP database: new developments. $\mathrm{Nu}$ cleic Acids Res., 36:D419-D425, 2008.

[5] A. F. Arenas, A. J. Gutierrez Escobar, and J. E. Gómez-Marin. Evolutionary origin of the protozoan parasites histone-like proteins (HU). In Silico Biology, 8:2, 2007.

[6] J. A. Armstrong. Negotiating the nucleosome: factors that allow RNA polymerase II to elongate through chromatin. Biochem Cell Biol, 85:426-434, 2007.

[7] T. A. Azam and A. Ishihama. Twelve species of the nucleoidassociated protein from Escherichia coli. sequence recognition specificity and DNA binding affinity. J. Biol. Chem. 274:33105-33113, 1999.

[8] K. A. Bailey, F. Marc, K. Sandman, and J. N. Reeve. Both DNA and histone fold sequences contribute to archaeal nucleosome stability. J Biol Chem, 277:9293-9301, 2002.

[9] K. A. Bailey, S. L. Pereira, J. Widom, and J. N. Reeve. Archaeal histone selection of nucleosome positioning sequences and the procaryotic origin of histone-dependent genome evolution. J Mol Biol, 303:25-34, 2000.

[10] J. Bailey-Serres, R. Sorenson, and P. Juntawong. Getting the message across: cytoplasmic ribonucleoprotein complexes. Trends Plant Sci., 14:443-453, 2009.

[11] A. J. Barrett and N. D. Rawlings. Evolutionary lines of cysteine peptidases. Biol Chem, 382:727-733, 2001.

[12] A. Basu, K. L. Rose, J. Zhang, R. C. Beavis, B. Ueberheide, B. A. Garcia, B. Chait, Y. Zhao, D. F. Hunt, E. Segal, C. D Allis, and S. B. Hake. Proteome-wide prediction of acetylation substrates. Proc Natl Acad Sci U S A, 106:13785-13790, 2009.

[13] A. Becerra, L. Delaye, S. Islas, and A. Lazcano. The very early stages of biological evolution and the nature of the Last Common Ancestor of the three major cell domains. Annual Review of Ecology, Evolution, and Systematics, 38:361-379, 2007.

[14] H. C. Beck, E. C. Nielsen, R. Matthiesen, L. H. Jensen, M. Sehested, P. Finn, M. Grauslund, A. M. Hansen, and O. N. Jensen. Quantitative proteomic analysis of post-translational modifications of human histones. Mol Cell Proteomics, 5:1314-1325, 2006.

[15] M. T. Bedford and S. G. Clarke. Protein arginine methylation in mammals: who, what, and why. Mol Cell., 33:1-13, 2009.

[16] M. T. Bedford and S. Richard. Arginine methylation an emerging regulator of protein function. Mol Cell, 18:263-272, 2005.

[17] S. D. Bell, C. H. Botting, B. N. Wardleworth, S. P. Jackson, and M. F. White. The interaction of Alba, a conserved archaeal chromatin protein, with $\mathrm{Sir} 2$ and its regulation by acetylation. Science, 296:148-151, 2002.
[18] A. Benecke. Chromatin code, local non-equilibrium dynamics, and the emergence of transcription regulatory programs. Eur Phys J E Soft Matter, 19:353-366, 2006.

[19] L. J. Benson, Y. Gu, T. Yakovleva, K. Tong, C. Barrows, C. L. Strack, R. G. Cook, C. A. Mizzen, and A. T. Annunziato. Modifications of $\mathrm{H} 3$ and $\mathrm{H} 4$ during chromatin replication, nucleosome assembly, and histone exchange. J Biol Chem., 281:9287-9396, 2006.

[20] P. G. Besant and P. V. Attwood. Detection of a mammalian histone $\mathrm{H} 4$ kinase that has yeast histidine kinase-like enzymic activity. Int J Biochem Cell Biol., 32:243-253, 2000.

[21] P. G. Besant and P. V. Attwood. Detection and analysis of protein histidine phosphorylation. Mol Cell Biochem., 329:93$106,2009$.

[22] S. R. Bhaumik, E. Smith, and A. Shilatifard. Covalent modifications of histones during development and disease pathogenesis. Nat Struct Mol Biol., 14:1008-1016, 2007.

[23] S. Bonn and E. E. Furlong. cis-regulatory networks during development: a view of Drosophila. Curr Opin Genet Dev., 18:513-520, 2008

[24] B. Boussau, S. Blanquart, A. Neculea, N. Lartillot, and M. Gouy. Parallel adaptations to high temperatures in the Archaean eon. Nature, 456:942-946, 2008.

[25] D. T. Brown. Histone variants: are they functionally heterogeneous? Genome Biol, 2, 2001.

[26] R. S. Brown. Zinc finger proteins: getting a grip on RNA. Curr Opin Struct Biol., 15:94-98, 2005.

[27] S. S. Broyles and D. E. Pettijohn. Interaction of the Escherichia coli $\mathrm{HU}$ protein with DNA. evidence for formation of nucleosome-like structures with altered DNA helical pitch J. Mol. Biol., 187:47-60, 1986.

[28] S. W. Buck, C. M. Gallo, and J. S. Smith. Diversity in the Sir2 family of protein deacetylases. J Leukoc Biol, 75:939-950, 2004.

[29] M. Bühler, A. Verdel, and D. Moazed. Tethering RITS to a nascent transcript initiates RNAi- and heterochromatindependent gene silencing. Cell, 125:873-886, 2006.

[30] S. W. Chan. Inputs and outputs for chromatin-targeted RNAi. Trends Plant Sci., 13:383-389, 2008.

[31] X. Cheng, R. E. Collins, and X. Zhang. Structural and sequence motifs of protein (histone) methylation enzymes. Annu Rev Biophys Biomol Struct, 34:267-294, 2005.

[32] N. Chomsky. On certain formal properties of grammars. In form. E Control, 2:137167, 1959.

[33] L. A. Cirillo, F. R. Lin, I. Cuesta, D. Friedman, M. Jarnik, and K. S. Zaret. Opening of compacted chromatin by early developmental transcription factors HNF3 (FoxA) and GATA4. Mol Cell, 9:279-289, 2002.

[34] L. Claret and J. Rouviere-Yaniv. Variation in HU composition during growth of Escherichia coli: the heterodimer is required for long term survival. J Mol Biol, 273:93-104, 1997.

[35] A. J. Cozzone. Bacterial tyrosine kinases: novel targets for antibacterial therapy? Trends Microbio., 17:536-543, 2009.

[36] L. Cubonová, K. Sandman, S. J. Hallam, E. F. Delong, and J. N. Reeve. Histones in crenarchaea. J Bacteriol, 187:5482-5485, 2005.

[37] G. Cuthbert, S. Daujat, A. W. Snowden, H. ErdjumentBromage, T. Hagiwara, M. Yamada, R. Schneider, P. D. Gregory, P. Tempst, A. J. Bannister, and T. Kouzarides. Histone deimination antagonizes arginine methylation. Cell, 118:545553,2004

[38] J. Dai and J. M. G. Higgins. Haspin: a mitotic histone kinase required for metaphase chromosome alignment. Cell Cycle, 4:665-668, 2005.

[39] R. T. Dame, M. Noom, and G. J. L. Wuite. Bacterial chromatin 
organization by H-NS protein unravelled using dual DNA manipulation. Nature, 444:387-390, 2006.

[40] X. de la Cruz, S. Lois, S. Sánchez-Molina, and M. A. MartínezBalbás. Do protein motifs read the histone code? Bioessays, 27:164-175, 2005

[41] M. Di Giulio. The origin of genes could be polyphyletic. Gene, 426:39-46, 2008.

[42] S. C. Dillon, X. Zhang, R. C. Trievel, and X. Cheng. The SETdomain protein superfamily: protein lysine methyltransferases. Genome Biol., 6:227, 2005.

[43] M. F. Dion, S. J. Altschuler, L. F. Wu, and O. J. Rando. Genomic characterization reveals a simple histone $\mathrm{H} 4$ acetylation code. Proc Natl Acad Sci U S A, 102:5501-5506, 2005.

[44] A. M. Dri, J. Rouviere-Yaniv, and P. L. Moreau. Inhibition of cell division in hupA/hupB mutant bacteria lacking HU protein. J. Bacteriol., 173:2852-2863, 1991.

[45] J. Eichler and M. W. Adams. Posttranslational protein modification in Archaea. Microbiol Mol Biol Rev, 69:393-425, 2005.

[46] M. Eigen. Selforganization of matter and the evolution of macromolecules. Naturwiss., 58:465-523, 1971.

[47] M. Eigen, J. McCaskill, and P. Schuster. The molecular quasispecies. Adv. Chem. Phys., 75:149-263, 1989.

[48] J. C. Eissenberg. Molecular biology of the chromo domain: an ancient chromatin module comes of age. Gene, 275:19-29, 2001.

[49] A. E. Escargueil, D. G. Soares, M. Salvador, A. K. Larsen, and J. A. Henriques. What histone code for DNA repair? Mutat Res., 658:259-270, 2008.

[50] M. Esteller and J. G. Herman. Cancer as an epigenetic disease: DNA methylation and chromatin alterations in human tumours. J Pathol., 196:1-7, 2002.

[51] R. J. Ferl, M. S. Manak, and M. F. Reyes. The 14-3-3s. Genome Biol, 3:7, 2002.

[52] W. Fischle, Y. Wang, and C. D. Allis. Binary switches and modification cassettes in histone biology and beyond. Nature, 425:475-479, 2003

[53] F. Forneris, C. Binda, M. A. Vanoni, E. Battaglioli, and A. Mattevi. Human histone demethylase LSD1 reads the histone code. J Biol Chem, 280:41360-41365, 2005.

[54] P. Forterre. The origin of DNA genomes and DNA replication proteins. Curr. Op. Microbiol., 5:525-532, 2002.

[55] P. Forterre. Three RNA cells for ribosomal lineages and three DNA viruses to replicate their genomes: A hypothesis for the origin of cellular domain. Proc. Natl. Acad. Sci. USA, 103:3669-3674, 2006

[56] M. Freitag and E. U. Selker. Controlling DNA methylation: many roads to one modification. Curr Opin Genet Dev, 15:191-199, 2005

[57] M. A. Freitas, A. R. Sklenar, and M. R. Parthun. Application of mass spectrometry to the identification and quantification of histone post-translational modifications. J Cell Biochem. 92:691-700, 2004

[58] I. Frenkiel-Krispin, D andBen-Avraham, J. Englander, E. Shimoni, S. G. Wolf, and A. Minsky. Nucleoid restructuring in stationary-state bacteria. Mol Microbiol., 51:395-405, 2004.

[59] R. A. Frye. Phylogenetic classification of prokaryotic and eukaryotic Sir2-like proteins. Biochem Biophys Res Commun, 273:793-798, 2000.

[60] B. A. Garcia, S. B. Hake, R. L. Diaz, M. Kauer, S. A. Morris, J. Recht, J. Shabanowitz, N. Mishra, B. D. Strahl, C. D. Allis, and D. F. Hunt. Organismal differences in post-translational modifications in histones H3 and H4. J Biol Chem, 282:7641$7655,2007$.

[61] N. Glansdorff, Y. Xu, and B. Labedan. The Last Universal Common Ancestor: emergence, constitution and genetic legacy of an elusive forerunner. Biology Direct, 3:29, 2008.

[62] R. A. Grayling, K. Sandman, and J. N. Reeve. Histones and chromatin structure in hyperthermophilic Archaea. FEMS Microbiol Rev., 18:203-213, 1996.

[63] I. V. Gregoretti, Y. M. Lee, and H. V. Goodson. Molecular evolution of the histone deacetylase family: functional implications of phylogenetic analysis. J Mol Biol, 338:17-31, 2004

[64] N. A. Grieshaber, E. R. Fischer, D. J. Mead, C. A. Dooley, and T. Hackstadt. Chlamydial histone-DNA interactions are disrupted by a metabolite in the methylerythritol phosphate pathway of isoprenoid biosynthesis. Proc Natl Acad Sci U S A, 101:7451-7456, 2004

[65] L. Guo, Y. Feng, Z. Zhang, H. Yao, Y. Luo, J. Wang, and L. Huang. Biochemical and structural characterization of Cren7, a novel chromatin protein conserved among Crenarchaea. Nucleic Acids Res, 36:1129-1137, 2008.

[66] S. B. Hake and C. D. Allis. Histone H3 variants and their potential role in indexing mammalian genomes: the "H3 barcode hypothesis". Proc Natl Acad Sci U S A, 103:6428-6435, 2006.

[67] I. M. Hall, G. D. Shankaranarayana, K. Noma, N. Ayoub, A. Cohen, and S. I. Grewal. Establishment and maintenance of a heterochromatin domain. Science, 297:2232-2237, 2002.

[68] T. M. Hall. Multiple modes of RNA recognition by zinc finger proteins. Curr Opin Struct Biol., 15:367-373, 2005.

[69] A. Hammet, C. Magill, J. Heierhorst, and S. P. Jackson. Rad9 BRCT domain interaction with phosphorylated $\mathrm{H} 2 \mathrm{AX}$ regulates the G1 checkpoint in budding yeast. EMBO Rep., 8:851$857,2007$.

[70] S. Healy, T. D. Heightman, L. Hohmann, D. Schriemer, and R. A. Gravel. Nonenzymatic biotinylation of histone H2A Protein Sci., 18:314-328, 2009.

[71] B. Hekimoglu and L. Ringrose. Non-coding RNAs in polycomb/trithorax regulation. RNA Biol., 6:129-137, 2009.

[72] S. Henikoff. Histone modifications: combinatorial complexity or cumulative simplicity? Proc Natl Acad Sci U S A, 102:5308-5309, 2005

[73] S. Henikoff. Nucleosome destabilization in the epigenetic regulation of gene expression. Nat Rev Genet, 9:15-26, 2008.

[74] M. Hochstrasser. Evolution and function of ubiquitin-like protein-conjugation systems. Nat Cell Biol., 2:E153-E157, 2000.

[75] M. Hochstrasser. Origin and function of ubiquitin-like proteins. Nature, 458:422-429, 2009.

[76] S. Istrail, S. B. De-Leon, and E. H. Davidson. The regulatory genome and the computer. Dev Biol., 310:187-195, 2007.

[77] L. M. Iyer, V. Anantharaman, M. Y. Wolf, and L. Aravind. Comparative genomics of transcription factors and chromatin proteins in parasitic protists and other eukaryotes. Int J Parasitol., 38:1-31, 2008.

[78] S. Izawa and Y. Inoue. Post-transcriptional regulation of gene expression in yeast under ethanol stress. Biotechnol Appl Biochem., 53:93-99, 2009.

[79] M. G. Izban and D. S. Luse. Factor-stimulated RNA polymerase II transcribes at physiological elongation rates on naked DNA but very poorly on chromatin templates. J Biol Chem, 267:13647-13655, 1992

[80] C. Jelinska, M. J. Conroy, C. J. Craven, A. M. Hounslow, P. A. Bullough, J. P. Waltho, G. L. Taylor, and M. F. White. Obligate heterodimerization of the archaeal Alba2 protein with Alba1 provides a mechanism for control of DNA packaging. Structure, 13:963-971, 2005.

[81] C. Jiang and B. F. Pugh. Nucleosome positioning and gene regulation: advances through genomics. Nat Rev Genet., 10:161$172,2009$.

[82] J. Jin, Y. Cai, B. Li, R. C. Conaway, J. L. Workman, J. W. 
Conaway, and T. Kusch. In and out: histone variant exchange in chromatin. Trends Biochem Sci., 30:680-687, 2005.

[83] R. T. Kamakaka and S. Biggins. Histone variants: deviants? Genes Dev, 19:295-310, 2005.

[84] H. E. Kasinsky, J. D. Lewis, J. B. Dacks, and J. Ausió. Origin of H1 linker histones. FASEB J., 15:34-42, 2001.

[85] P. J. Kennely. Protein kinases and protein phosphatases in prokaryotes: a genomic perspective. FEMS Microbiol Lett., 206:1-8, 2002.

[86] P. J. Kennely. Archaeal protein kinases and protein phosphatases: insights from genomics and biochemistry. Biochem J., 370:373-389, 2003.

[87] A. M. Khalil, M. Guttman, M. Huarte, M Garber, A. Raj, D. Rivea Morales, K. Thomas, A. Presser, B. E. Bernstein, A. van Oudenaarden, A. Regev, E. S. Lander, and J. L. Rinn. Many human large intergenic noncoding RNAs associate with chromatin-modifying complexes and affect gene expression. Proc. Natl. Acad. Sci. USA, 106:11675-11680, 2009.

[88] M. S. Klenov, S. A. Lavrov, A. D. Stolyarenko, S. S. Ryazansky, A. A. Aravin, T. Tuschl, and V. A. Gvozdev. Repeatassociated siRNAs cause chromatin silencing of retrotransposons in the Drosophila melanogaster germline. Nucleic Acids Res., 35:5430-5438, 2007.

[89] E. V. Koonin and W. Martin. On the origin of genomes and cells within inorganic compartments. Trends Genet., 21:647654, 2005.

[90] N. Kothapalli, G. Camporeale, A. Kueh, Y. C. Chew, A. M. Oommen, J. B. Griffin, and J. Zempleni. Biological functions of biotinylated histones. J Nutr Biochem., 16:446-448, 2005.

[91] G. Koutroubas, M. Merika, and D. Thanos. Bypassing the requirements for epigenetic modifications in gene transcription by increasing enhancer strength. Mol Cell Biol, 28:926-938, 2008.

[92] F. L. Kuang, Z. Luo, and M. D. Scharff. H3 trimethyl K9 and $\mathrm{H} 3$ acetyl $\mathrm{K} 9$ chromatin modifications are associated with class switch recombination. Proc Natl Acad Sci USA, 106:52885293, 2009.

[93] H. M. Kurth and K. Mochizuki. Non-coding RNA: A bridge between small RNA and DNA. RNA Biol., 6:138-140, 2009.

[94] M. Leeb, P. A. Steffen, and A. Wutz. X chromosome inactivation sparked by non-coding RNA. RNA Biol., 6:94-99, 2009.

[95] A. Lesne. The chromatin regulatory code: beyond a histone code. Eur Phys J E Soft Matter, 19:375-377, 2006.

[96] M. Levine and E. H. Davidson. Gene regulatory networks for development. Proc Natl Acad Sci USA, 102:4936-4942, 2005.

[97] B. Li, M. Carey, and J. L. Workman. The role of chromatin during transcription. Cell, 128:707-719, 2007.

[98] Y. Liu, S. D. Taverna, T. L. Muratore, J. Shabanowitz, D. F. Hunt, and C. D. Allis. RNAi-dependent H3K27 methylation is required for heterochromatin formation and DNA elimination in Tetrahymena. Genes Dev, 21:1530-1545, 2007.

[99] S. Lomvardas and D. Thanos. Opening chromatin. Mol Cell, 9:209-211, 2002.

[100] K. S. Long, J. Poehlsgaard, C. Kehrenberg, S. Schwarz, and B. Vester. The Cfr rRNA methyltransferase confers resistance to phenicols, lincosamides, oxazolidinones, pleuromutilins, and streptogramin A antibiotics. Antimicrob. Agents Chemother, 50:2500-2505, 2006.

[101] P. T. Lowary and J. Widom. New DNA sequence rules for high affinity binding to histone octamer and sequence-directed nucleosome positioning. J Mol Biol, 276:19-42, 1998.

[102] I. Lozada-Chávez, S. Chandra Janga, and J. Collado-Vides. Bacterial regulatory networks are extremely flexible in evolution. Nucleic Acids Res., 34:3434-3445, 2006.

[103] S. Lucchini, G. Rowley, M. D. Goldberg, D. Hurd, M. Har- rison, and J. C. D. Hinton. H-NS mediates the silencing of laterally acquired genes in bacteria. PLoS Pathogens, 2:e81, 2006.

[104] M. S. Luijsterburg, M. F. White, R. van Driel, and R. T. Dame. The major architects of chromatin: architectural proteins in bacteria, archaea and eukaryotes. Crit Rev Biochem Mol Biol., 43:393-418, 2008.

[105] H. S. Malik and S. Henikoff. Phylogenomics of the nucleosome. Nat Struct Biol, 10:882-891, 2003.

[106] F. Marc, K. Sandman, R. Lurz, and J. N. Reeve. Archaeal histone tetramerization determines DNA affinity and the direction of DNA supercoiling. J Biol Chem, 277:30879-30886, 2002.

[107] V. L. Marsh, S. Y. Peak-Chew, and S. D. Bell. Sir2 and the acetyltransferase, Pat, regulate the archaeal chromatin protein, Alba. J Biol Chem, 280:21122-21128, 2005.

[108] C. Martens, K. Vandepoele, and Y. Van de Peer. Wholegenome analysis reveals molecular innovations and evolutionary transitions in chromalveolate species. Proc Natl Acad Sci USA, 105:3427-3432, 2008.

[109] W. K. Mat, H. Xue, and J. T. Wong. The genomics of LUCA Front Biosci., 13:5605-5613, 2008.

[110] J. S. Mattick. A new paradigm for developmental biology. $J$ Exp Biol, 210:1526-1547, 2007.

[111] J. S. Mattick, P. P. Amaral, M. E. Dinger, T. R. Mercer, and M. F. Mehler. RNA regulation of epigenetic processes. Bioessays, 31:51-59, 2009.

[112] M. Matzke, T. Kanno, L. Daxinger, B. Huettel, and A. J. Matzke. RNA-mediated chromatin-based silencing in plants. Curr Opin Cell Biol., 21:367-376, 2009.

[113] E. L. Mersfelder and M. R. Parthun. The tale beyond the tail: histone core domain modifications and the regulation of chromatin structure. Nucleic Acids Res., 34:2653-2662, 2006

[114] D. Moazed. Small RNAs in transcriptional gene silencing and genome defence. Nature, 457:413-420, 2009

115] S. Moreno Díaz de la Espina, E. Alverca, A. Cuadrado, and $\mathrm{S}$. Franca. Organization of the genome and gene expression in a nuclear environment lacking histones and nucleosomes: the amazing dinoflagellates. Eur J Cell Biol., 84:137-149, 2005.

[116] H. D. Morgan, F. Santos, K. Green, W. Dean, and W. Reik. Epigenetic reprogramming in mammals. Hum Mol Genet, 14(Spec No 1):47-58, 2005.

[117] D. K. Morrison. The 14-3-3 proteins: integrators of diverse signaling cues that impact cell fate and cancer development. Trends Cell Biol., 19:16-23, 2009.

[118] S. Mujtaba, L. Zeng, and M. M. Zhou. Structure and acetyllysine recognition of the bromodomain. Oncogene, 26:55215527, 2007.

[119] M. Murata, Y. Azuma, K. Miura, M. A. Rahman, M. Matsutani, M. Aoyama, H. Suzuki, K. Sugi, and M. Shirai. Chlamydial SET domain protein functions as a histone methyltransferase. Microbiology, 153:585-592, 2007.

[120] C. J. Nelson, H. Santos-Rosa, and T. Kouzarides. Proline isomerization of histone $\mathrm{H} 3$ regulates lysine methylation and gene expression. Cell, 126:905-916, 2006.

[121] M. J. Nemeth and C. H. Lowrey. An erythroid-specific chromatin opening element increases beta-globin gene expression from integrated retroviral gene transfer vectors. Gene Ther Mol Biol., 8:475-486, 2004.

[122] R. K. Ng and J. B. Gurdon. Epigenetic inheritance of cell differentiation status. Cell Cycle, 7:1173-1777, 2008.

[123] S. M. Nijman, M. P. Luna-Vargas, A. Velds, T. R. Brummelkamp, A. M. Dirac, T. K. Sixma, and R. Bernards. A genomic and functional inventory of deubiquitinating enzymes. Cell, 123:773-786, 2005.

[124] M. C. Noom, F. J. H. Hol, N. Laurens, M. F. While, R. T. Dame, 
and G. J. L. Wuite. Unravelling the role of Alba in the organization of the archaeal nucleoid. Biophys. J., 96:Supplement 1, 61a, 2009. Poster Abstract.

[125] V. Norris. Poly-(R)-3-hydroxybutyrate and the pioneering work of Rosetta Natoli Reusch. Cell Mol Biol, 51:629-634, 2005.

[126] F. Orfaniotou, P. Tzamalis, A. Thanassoulas, E. Stefanidi, A. Zees, E. Boutou, M. Vlassi, G. Nounesis, and C. E. Vorgias The stability of the archaeal HU histone-like DNA-binding protein from Thermoplasma volcanium. Extremophiles, 13:110, 2009.

[127] R. Pandey, A. Müller, C. A. Napoli, D. A. Selinger, C. S. Pikaard, E. J. Richards, J. Bender, D. W. Mount, and R. A. Jorgensen. Analysis of histone acetyltransferase and histone deacetylase families of Arabidopsis thaliana suggests functional diversification of chromatin modification among multicellular eukaryotes. Nucleic Acids Res, 30:5036-5055, 2002.

[128] R. R. Pandey, T. Mondal, F. Mohammad, S. Enroth, L. Redrup, J. Komorowski, T. Nagano, D. Mancini-Dinardo, and C. Kanduri. Kcnq1ot1 antisense noncoding RNA mediates lineagespecific transcriptional silencing through chromatin-level regulation. Mol Cell., 32:232-246, 2008.

[129] T. Pélissier and M. Wassenegger. A DNA target of $30 \mathrm{bp}$ is sufficient for RNA-directed DNA methylation. RNA, 6:55-65, 2000.

[130] J. C. Perez, T. Latifi, and E. A. Groisman. Overcoming HNS-mediated transcriptional silencing of horizontally acquired genes by the PhoP and SlyA proteins in Salmonella enterica. $J$ Biol Chem, 283:10773-10783, 2008.

[131] J. Pérez-Martín and V. de Lorenzo. Clues and consequences of DNA bending in transcription. Аnпи Rev Microbiol, 51:593628,1997

[132] V. Pinson, M. Takahashi, and J. Rouviere-Yaniv. Differential binding of the escherichia coli $\mathrm{HU}$, homodimeric forms and heterodimeric form to linear, gapped and cruciform DNA. $J$. Mol. Biol, 267:485-497, 1999.

[133] A. M. Poole and D. T. Logan. Modern mRNA proofreading and repair: clues that the last universal ancestor possessed an RNA genome? Mol. Biol. Evol., 22:1444-1455, 2005.

[134] A. V. Probst, E. Dunleavy, and G. Almouzni. Epigenetic inheritance during the cell cycle. Nat Rev Mol Cell Biol., 10:192206, 2009.

[135] S. J. Prohaska and P. F. Stadler. A story of growing confusion: Genes and their regulation. In R. P. Mondaini and R. Dilão, editors, BIOMAT-2007: International Symposium on Mathematical and Computational Biology, pages 325-345, Singapore, 2007. World Scientific. Armação dos Búzios, RJ, Brazil, 2429 November 2008

[136] O. J. Rando and K. Ahmad. Rules and regulation in the primary structure of chromatin. Curr Opin Cell Biol, 19:250-256, 2007.

[137] J. N. Reeve, K, A. Bailey, W. T. Li, F. Marc, K. Sandman, and D. J. Soares. Archaeal histones: structures, stability and DNA binding. Biochem Soc Trans, 32:227-230, 2004.

[138] W. Reik. Stability and flexibility of epigenetic gene regulation in mammalian development. Nature, 447:425-432, 2007.

[139] J. L. Rinn, M. Kertesz, J. K. Wang, S. L. Squazzo, X. Xu, S. A. Brugmann, L. H. Goodnough, J. A. Helms, P. J. Farnham, E. Segal, and H. Y. Chang. Functional demarcation of active and silent chromatin domains in human HOX loci by noncoding RNAs. Cell, 29:1311-1323, 2007.

[140] F. Rodríguez-Trelles, R. Rosa Tarrío, and F. J. Ayala. Origins and evolution of spliceosomal introns. Annu. Rev. Genet. 40:47-76, 2006

[141] T. Y. Roh, S. Cuddapah, and K. Zhao. Active chromatin do- mains are defined by acetylation islands revealed by genomewide mapping. Genes Dev, 19:542-552, 2005.

[142] S. E. Rundlett, A. A. Carmen, R. Kobayashi, S. Bavykin, B. M Turner, and M. Grunstein. HDA1 and RPD3 are members of distinct yeast histone deacetylase complexes that regulate silencing and transcription. Proc Natl Acad Sci US A, 93:1450314508, 1996.

[143] M. P. Ryan, R. Jones, and R. H. Morse. SWI-SNF complex participation in transcriptional activation at a step subsequent to activator binding. Mol Cell Biol, 18:1774-1782, 1998.

[144] T. Sanchez-Elsner, D. Gou, E. Kremmer, and F. Sauer. Noncoding RNAs of trithorax response elements recruit Drosophila Ash1 to Ultrabithorax. Science, 311:1118-1123, 2006.

[145] K. Sandman, R. A. Grayling, B. Dobrinski, R. Lurz, and J. A. Reeve. Growth-phase-dependent synthesis of histones in the archaeon Methanothermus fervidus. Proc. Natl. Acad. Sci. USA, 91:12624-12628, 1994.

[146] K. Sandman, S. L. Pereira, and J. N. Reeve. Diversity of prokaryotic chromosomal proteins and the origin of the nucleosome. Cell Mol Life Sci, 54:1350-1364, 1998.

[147] K. Sandman and J. N. Reeve. Structure and functional relationships of archaeal and eukaryal histones and nucleosomes. Arch Microbiol, 173:165-169, 2000.

[148] D. Schübeler, C. Francastel, D. M. Cimbora, A. Reik, D. I. Martin, and M. Groudine. Nuclear localization and histone acetylation: a pathway for chromatin opening and transcriptional activation of the human beta-globin locus. Genes Dev, 14:940-950, 2000

[149] H. L. Schubert, R. M. Blumenthal, and X. Cheng. Many paths to methyltransfer: a chronicle of convergence. Trends Biochem Sci, 28:329-335, 2003.

[150] B. Schuettengruber, D. Chourrout, M. Vervoort, B. Leblanc, and G. Cavalli. Genome regulation by polycomb and trithorax proteins. Cell, 128:735-745, 2007.

[151] Y. B. Schwartz and V. Pirrotta. Polycomb complexes and epigenetic states. Curr Opin Cell Biol., 20:266-273, 2008.

[152] M. Sedighi and A. M. Sengupta. Epigenetic chromatin silencing: bistability and front propagation. Phys Biol, 4:246-255, 2007.

[153] E. Segal and J. Widom. What controls nucleosome positions? Trends Genet., 25:225-343, 2009.

[154] Y. Shi and J. M. Berg. Specific DNA-RNA hybrid binding by zinc finger proteins. Science, 268:282-284, 1995.

[155] Y. Shi, F. Lan, C. Matson, P. Mulligan, J. R. Whetstine, P. A. Cole, R. A. Casero, and Y. Shi. Histone demethylation mediated by the nuclear amine oxidase homolog LSD1. Cell, 119:941-953, 2004.

[156] T. N. Siegel, D. R. Hekstra, L. E. Kemp, L. M. Figueiredo, J. E. Lowell, D. Fenyo, X. Wang, S. Dewell, and G. A. Cross. Four histone variants mark the boundaries of polycistronic transcription units in Trypanosoma brucei. Genes Dev., 23:1063-1076, 2009.

[157] S. K. Singh, S. R. Choudhury, S. Roy, and D. N. Sengupta. Sequential, structural, and phylogenetic study of BRCT module in plants. J Biomol Struct Dyn., 26:235-245, 2008.

[158] A. I. Slesarev, G. I. Belova, S. A. Kozyavkin, and J. A. Lake. Evidence for an early prokaryotic origin of histones $\mathrm{H} 2 \mathrm{~A}$ and H4 prior to the emergence of eukaryotes. Nucleic Acids Res, 26:427-430, 1998 .

[159] J. S. Smith, C. B. Brachmann, I. Celic, M. A. Kenna, S. Muhammad, V. J. Starai, J. L. Avalos, J. C. EscalanteSemerena, C. Grubmeyer, C. Wolberger, and J. D. Boeke. A phylogenetically conserved NAD+-dependent protein deacetylase activity in the Sir2 protein family. Proc Natl Acad Sci U S A, 97:6658-6663, 2000. 
[160] V. J. Starai and J. C. Escalante-Semerena. Identification of the protein acetyltransferase (Pat) enzyme that acetylates acetylCoA synthetase in Salmonella enterica. J Mol Biol, 340:10051012, 2004.

[161] W. Sun, X. Xu, M. Pavlova, A. M. Edwards, A. Joachimiak, A. Savchenko, and D. Christendat. The crystal structure of a novel SAM-dependent methyltransferase PH1915 from Pyrococcus horikoshii. Protein Sci, 14:3121-3128, 2005.

[162] P. B. Talbert and S. Henikoff. Chromatin-based transcriptional punctuation. Genes Dev., 23:1037-1041, 2009.

[163] E. Tan, P. G. Besant, X. L. Zu, C. W. Turck, M. A. Bogoyevitch, S. G. Lim, P. V. Attwood, and G. C. Yeoh. Histone H4 histidine kinase displays the expression pattern of a liver oncodevelopmental marker. Carcinogenesis, 25:2083-2088, 2004.

[164] M. Tariq and J. Paszkowski. DNA and histone methylation in plants. Trends Genet, 20:244-251, 2004.

[165] J. Taunton, C. A. Hassig, and S. L. Schreiber. A mammalian histone deacetylase related to the yeast transcriptional regulator Rpd3p. Science, 272:408-411, 1996.

[166] S. D. Taverna, H. Li, A. J. Ruthenburg, C. D. Allis, and D. J. Patel. How chromatin-binding modules interpret histone modifications: lessons from professional pocket pickers. Nat Struct Mol Biol, 14:1025-1040, 2007.

[167] B. L. Taylor. An alternative strategy for adaptation in bacterial behavior. J Bacteriol, 186:3671-3673, 2004.

[168] W. R. Taylor. Transcription and translation in an RNA world. Phil. Trans. R. Soc. B, 361:1751-1760, 2006.

[169] M. Thanbichler, S. C. Wang, and L. Shapiro. The bacterial nucleoid: A highly organized and dynamic structure. J. Cell. Biochem., 96:506-521, 2005.

[170] T. H. Thatcher and M. A. Gorovsky. Phylogenetic analysis of the core histones H2A, H2B, H3, and H4. Nucleic Acids Res, 22:174-179, 1994

[171] M. E. Torres-Padilla, D. E. Parfitt, T. Kouzarides, and M. Zernicka-Goetz. Histone arginine methylation regulates pluripotency in the early mouse embryo. Nature, 445:214-218, 2007.

[172] A. Travers and G. Muskhelishvili. Bacterial chromatin. Cur. Op. Genet. Devel., 15:507-514, 2005

[173] S. C. Trewick, P. J. McLaughlin, and R. C. Allshire. Methylation: lost in hydroxylation? EMBO Rep, 6:315-320, 2005

[174] O. Triana, N. Galanti, N. Olea, U. Hellman, C. Wernstedt, H. Lujan, C. Medina, and G. C. Toro. Chromatin and histones from Giardia lamblia: a new puzzle in primitive eukaryotes. $J$ Cell Biochem., 82:573-582, 2001.

[175] Y. Tsukada, J. Fang, H. Erdjument-Bromage, M. E. Warren, C. H. Borchers, P. Tempst, and Y. Zhang. Histone demethylation by a family of JmjC domain-containing proteins. Nature, 439:811-816, 2006.

[176] A. Verdel, A. Vavasseur, M. Le Gorrec, and L. TouatTodeschini. Common themes in siRNA-mediated epigenetic silencing pathways. Int J Dev Biol., 53:245-257, 2009.

[177] M. W. Vetting, S. Magnet, E. Nieves, S. L. Roderick, and J. S. Blanchard. A bacterial acetyltransferase capable of regioselective $\mathrm{N}$-acetylation of antibiotics and histones. Chem Biol, 11:565-573, 2004

[178] G. Vilkaitis, I. Suetake, S. Klimasauskas, and S. Tajima. Processive methylation of hemimethylated $\mathrm{CpG}$ sites by mouse Dnmt1 DNA methyltransferase. J Biol Chem., 280:64-72, 2005 .

[179] H. Wang, L. Zhai, J. Xu, H.-Y. Joo, S. Jackson, H. ErdjumentBromage, P. Tempst, Y. Xiong, and Y. Zhang. Histone $\mathrm{H}_{3}$ and $\mathrm{H} 4$ ubiquitylation by the CUL4-DDB-ROC1 ubiquitin ligase facilitates cellular response to DNA damage. Mol. Cell,
$22 \cdot 383-394,2006$

[180] Y. Wang, J. Wysocka, J. Sayegh, Y. H. Lee, J. R. Perlin, L. Leonelli, L. S. Sonbuchner, C. H. McDonald, R. G. Cook, Y. Dou, R. G. Roeder, S. Clarke, M. R. Stallcup, C. D. Allis, and S. A. Coonrod. Human PAD4 regulates histone arginine methylation levels via demethylimination. Science, 306:279 283,2004

[181] B. N. Wardleworth, R. J. Russell, S. D. Bell, G. L. Taylor, and M. F. White. Structure of alba: an archaeal chromatin protein modulated by acetylation. EMBO J, 21:4654-4662, 2002.

[182] S. Washietl, R. Machné, and N. Goldman. Evolutionary footprints of nucleosome positions in yeast. Trends Genet. 24:583-587, 2008

[183] V. M. Weake and J. L. Workman. Histone ubiquitination: Triggering gene activity. Mol. Cell, 28:653-663, 2008.

[184] D. Wenkert and C. D. Allis. Timing of the appearance of macronuclear-specific histone variant hv1 and gene expression in developing new macronuclei of Tetrahymena thermophila. $J$ Cell Biol, 98:2107-2117, 1984.

[185] M. F. White and S. D. Bell. Holding it together: chromatin in the archaea. Trends Genet, 18:621-626, 2002.

[186] D. Wilson, R. Pethica, Y. Zhou, C. Talbot, C. Vogel, M. Madera, C. Chothia, and J. Gough. SUPERFAMILY comparative genomics, datamining and sophisticated visualisation. Nucleic Acids Res., 37:D380-D386, 2009

[187] Y. I. Wolf and E. V. Koonin. On the origin of the translation system and the genetic code in the RNA world by means of natural selection, exaptation, and subfunctionalization. Biology Direct, 2:14, 2007

[188] J. T. Y. Wong, D. C. New, J. C. W. Wong, and V. K. L. Hung. Histone-like proteins of the dinoflagellate Crypthecodinium cohnii have homologies to bacterial DNA-binding proteins. Eukaryotic Cell, 2:646-650, 2003.

[189] C. Xu, G. Cui, M. V. Botuyan, and G. Mer. Structural basis for the recognition of methylated histone H3K36 by the Eaf3 subunit of histone deacetylase complex Rpd3S. Structure, 16:1740-1750, 2008 .

[190] J. Yang, S. Medvedev, J. Yu, R. M. Schultz, and N. B. Hecht Deletion of the DNA/RNA-binding protein MSY2 leads to post-meiotic arrest. Mol Cell Endocrinol., 250:20-24, 2006.

[191] H. S. Yoon, J. D. Hackett, G. Pinto, and D. Bhattacharya. The single, ancient origin of chromist plastids. Proc Natl Acad Sci USA, 99:15507-15512, 2002

[192] L. Zhang, E. E. Eugeni, M. R. Parthun, and M. A. Freitas. Identification of novel histone post-translational modifications by peptide mass fingerprinting. Chromosoma, 112:77-86, 2003.

[193] L. Zhang, K. Jones, and F. Gong. The molecular basis of chromatin dynamics during nucleotide excision repair. Biochem Cell Biol., 87:265-272, 2009.

[194] W. Zhou, X. Wang, and M. G. Rosenfeld. Histone H2A ubiquitination in transcriptional regulation and DNA damage repair Int J Biochem Cell Biol., 41:12-15, 2009. 